This is page 1

Printer: Opaque this

\title{
Disordered Quantum Solids
}

\section{T. Giamarchi, E. Orignac}

\begin{abstract}
Due to the peculiar non-fermi liquid of one dimensional systems, disorder has particularly strong effects. We show that such systems belong to the more general class of disordered quantum solids. We discuss the physics of such disordered interacting systems and the methods that allows to treat them. In addition to, by now standard renormalization group methods, We explain how a simple variational approach allows to treat these problems even in case when the RG fails. We discuss various physical realizations of such disordered quantum solids both in one and higher dimensions (Wigner crystal, Bose glass). We investigate in details the interesting example of a disordered Mott insulator and argue that intermediate disorder can lead to a novel phase, the Mott glass, intermediate between a Mott and and Anderson insulator.
\end{abstract}

\section{Introduction}

Disorder effects are omnipresent in condensed matter physics, where one has to struggle very hard to deal with clean systems. Quite remarkably fermionic system exhibit marked differences with disorder in classical systems. Indeed the very existence of a Fermi energy $E_{F}$ "reduces" the effects of disorder since the relevant parameter now become the relative strength of the disorder compared to the Fermi energy $D / E_{F}$ or the mean free path compared to the Fermi length $k_{F} l$. Of course nature would not remain as simple as that, and quantum effects lead in fact also to reinforcement of the disorder effects and turn in low dimension a free electron system into an insulator, as pointed out by Mott and Twose国.

We have now gained a very good understanding of the properties of such disordered free electron systems. To tackle them an arsenal of methods ranging from diagrams [2] 3], scaling theory [4], replicas [5, 6], supersymmetry [7] have been developped. Life become much less simple when interactions among fermions are taken into account. Although it is very intuitive to think that when interactions are small the noninteracting problem is a good starting point, such an intuition turns out to be wrong for a number of reasons: (i) even if in the pure system interactions can be "removed" from the system by resorting to Fermi Liquid theory [8] this is not the case when disorder is present. Because disorder renders electrons slowly diffusive slowly rather than ballistic, they feel the interactions much more strongly with explosive results [9]. Effective interactions increase when looking at low en- 
ergy properties and Fermi liquid theory breaks down. The non-interacting physics is thus only relevant for very low disorder. (ii) When the dimension is small or the interactions strong to start with (like in systems undergoing Mott transitions) it is of course impossible to start from the non-interacting limit and one has to solve the full problem. What puts us at a disadvantage here is that most of the techniques useful for the noninteracting case also fail as soon as interactions are included: (i) supersymmetric method, which rests by construction on the quadratic nature of the hamiltonian is useless. (ii) it is now difficult now to really determine classes of diagram to sum.

Disorder can still be averaged over by using replicas but then one is left with a complicated (and untractable) theory. Renormalisation group attempts have been made with some success 10, 11] but also with the problem that the coupling constants diverge, so that the low energy fixed point remains elusive. Many other attempts in treating this very complicated problem exist in the literature and it is impossible to list them all[12. Note that numerical studies are also hampered when studying this problem both because of the difficulty in taking the interactions into account (which more or less imposes either Monte-Carlo or exact diagonalization) and then to perform the complicated disorder averaging with enough statistics or large system sizes to get reliable results.

A very peculiar situation occurs when one considers one dimension. On one hand one expects the difficulty to be maximum here. The interactions lead to very strong effects and destroy any trace of Fermi liquid giving rise to what is known as a Lutinger liquid. The disorder is also extremely strong, giving rise for the noninteracting case to a system so localized that the localisation length is simply the mean free path and a diffusive regime is absent. On the other hand one is in $d=1$ in a much better situation to tackle the problem since the interactions can be treated essentially exactly using for example techniques such as bosonization 13, 14, 15, 16, 17 so one only needs good techniques to tackle the disorder. Quite interestingly the physics of interacting and disordered one dimensional systems is the one of disordered quantum solids. Higher dimensional examples of such systems are the Wigner crystal, Charge Density Waves and Bose glass. In these notes, we will explain the techniques allowing to treat such systems, ranging from simple physical arguments to a quite sophisticated variational approach. In order to remain pedagogical and keep the algegra simple we mostly discuss the technicalities on the simplest example of spinless fermions. We briefly discuss the specific physical realizations and give references so that the reader can look in more details the physical properties of these specific systems.

Before we embark with the physics, let us point out that these notes results from the synthesis of various lectures. Some arbitrary choice of material had to be made in order to keep some level of clarity. Even if we have made some effort to cover various interesting topics, these notes cannot pretend to be as exhaustive as a full review. We thus apologize in advance 
to anybody whose pet problem (or paper) is not covered in these few pages.

\section{Disordered interacting Fermions}

\subsection{Model}

Let us consider spinless fermions hopping on a lattice with a kinetic energy $t$ and an interaction $V$

$$
\begin{aligned}
H & =-t \sum_{i}\left(c_{i}^{\dagger} c_{i+1}+h . c .\right) \\
& +\sum_{i>j} V_{i-j}\left(n_{i}-\bar{n}\right)\left(n_{j}-\bar{n}\right)
\end{aligned}
$$

where $n_{i}(\bar{n})$ is the local (average) electron density, and the rest of the notation is standard ( $t=1$ is the unit of energy). For nearest neighbor interactions this is the well known $t-V$ model. When using a JordanWigner transformation 18 to express the fermions in term of spins this latter model maps to an XXZ spin chain. In addition to (1.1) we want to submit the fermions to a disorder. We concentrate here on site disorder. Again most results/methods will carry over for randomness in the hopping. The randomness is simply

$$
H_{\mathrm{int}}=\sum_{i} \mu_{i}\left(n_{i}-\bar{n}\right)
$$

where $\mu_{i}$ is a random variable of zero mean.

\subsection{Pure system}

The low energy properties of the pure system (1.1) are by now well understood. We will thus review the bosonization of (1.1) only very briefly to fix the notations and refer the reader to the various reviews on the subject [13, 14, 15, 16, 17]. To get the low energy physics, it is enough to focus on the excitations aroung the Fermi points. In the continuum limit $i \rightarrow x$ this

amounts to express the fermion field in term of slow varying (with respect to the lattice spacing) field of right (with momentum close to $+k_{F}$ ) and left (with momentum close to $-k_{F}$ ) movers

$$
c_{i}^{\dagger} \rightarrow \psi^{\dagger}(x)=e^{-i k_{F} x} \psi_{+}^{\dagger}(x)+e^{i k_{F} x} \psi_{-}^{\dagger}(x)
$$

In term of these fields 1.1 ) becomes

$H=-i \hbar v_{F} \int d x\left(\psi_{+}^{\dagger} \partial_{x} \psi_{+}(x)-\psi_{-}^{\dagger} \partial_{x} \psi_{-}(x)\right)+\int d x_{1} d x_{2} V\left(x_{1}-x_{2}\right) \rho\left(x_{1}\right) \rho\left(x_{2}\right)$ 
where the density reads

$$
\rho(x)=\psi_{+}^{\dagger} \psi_{+}+\psi_{-}^{\dagger} \psi_{-}+\left(e^{-i 2 k_{F} x} \psi_{+}^{\dagger} \psi_{-}+\text {h.c. }\right)
$$

The remarkable feature in $d=1$ is that all the excitations of the system can be reexpressed in term of the fluctuations of density. If one introduces a field $\phi(x)$ describing the long wavelength part of the density (1.5) reads

$$
\rho(x)=\rho_{0}-\frac{1}{\pi} \nabla \phi(x)+\frac{1}{(2 \pi \alpha)}\left(e^{-i 2 k_{F} x+2 \phi(x)}+\text { h.c. }\right)
$$

where $\alpha$ is a lattice spacing, $\rho_{0}=\bar{n} / \alpha$ and:

$$
\begin{aligned}
\nabla \phi(r) & =\sum_{ \pm} \psi_{ \pm}^{\dagger}(x) \psi_{ \pm}(x) \\
\nabla \theta(r) & =\sum_{ \pm} \pm \psi_{ \pm}^{\dagger}(x) \psi_{ \pm}(x)
\end{aligned}
$$

$\theta$ is a similar field but associated with the long wavelength part of the current. $\phi$ and $\Pi=\frac{1}{\pi} \nabla \theta$ are canonically conjugate. Both the Hamiltonian and the fermion operator can be expressed in terms of these two fields

$$
\begin{aligned}
H & =\frac{1}{2 \pi} \int d x\left[u K(\nabla \theta(x))^{2}+\frac{u}{K}(\nabla \phi(x))^{2}\right] \\
\psi_{ \pm}(r) & =\frac{1}{\sqrt{2 \pi \alpha}} e^{-i( \pm \phi(r)-\theta(r))}
\end{aligned}
$$

Using (1.10) in (1.5) one easily recovers (1.6). For the free Hamiltonian (1.1) (with $V=0$ ) one has in (1.9) $u=v_{F}$ and $K=1$. What makes the boson representation so useful is the fact that even in the presence of interactions the bosonized form of (1.1) remains (1.9) but with renormalized (Luttinger Liquid) parameters $u$ and $K$.

\subsection{Disorder}

Similarly the disorder can be rewritten in the boson representation. It is natural to separate the Fourier components with wavevectors close to $q \sim 0$ $(\eta)$ and $q \sim \pm 2 k_{F}\left(\xi, \xi^{*}\right)$. The disorder becomes thus

$$
H_{\text {dis }}=\eta(x)\left[\psi_{+}^{\dagger} \psi_{+}+\psi_{-}^{\dagger} \psi_{-}\right]+\left(\xi(x) \psi_{+}^{\dagger} \psi_{-}+\text {h.c. }\right)
$$

where $\eta$ and $\xi$ are now two independent random potentials. $\eta$ is real but when the system is incommensurate $\left(2 k_{F} \neq \pi\right) \xi$ is complex. The potentials $\eta$ and $\xi$ correspond respectively to the forward scattering and the backward scattering of the fermions on impurities. (1.11) reads in the boson representation

$$
H_{\mathrm{dis}}=-\eta(x) \frac{1}{\pi} \nabla \phi(x)+\left[\frac{\xi(x)}{(2 \pi \alpha)} e^{i 2 \phi(x)}+\text { h.c. }\right]
$$


For incommensurate systems the forward scattering can easily be absorbed by a gauge transformation on the fermions. In the boson language this amounts to shift $\phi \rightarrow \phi-\frac{2 K}{u} \int^{x} d y \eta(y)$. Since $\xi$ is complex and hence has a random phase this shift does not affect the backscattering term (this will be even more explicit on the replicated Hamiltonian (1.46) below). Thus the forward scattering can be accounted for completely. Its main effect is to lead to an exponential decay of the disorder averaged density correlation functions. Since the current and the superconducting correlation functions depends only on $\theta$ (or equivalently in an action representation on $\partial_{t} \phi$ ) they are not affected by the shift. Transport properties thus depends on the backscattering alone, as is obvious on physical basis since forward scattering cannot change the current. For commensurate potentials the situation is mode complicated since $\xi$ is real $\left(e^{+i 2 k_{F}}=e^{-i 2 k_{F}}\right)$ and the forward scattering now does affect the backscattering term (in other words by combining a forward scattering and a scattering on the lattice one can generate a backscattering term). This has consequences that we will examine in more details in section 0 . For the moment let us focus on the incommensurate case and get rid of the forward scattering.

\section{Tackling the disorder}

Disordered one dimensional particles are thus described by

$$
S / \hbar=\int d x d \tau\left[\frac{1}{2 \pi K}\left[\frac{1}{v}\left(\partial_{\tau} \phi\right)^{2}+v\left(\partial_{x} \phi\right)^{2}\right]+\frac{\xi(x)}{2 \pi \alpha \hbar} e^{i 2 \phi(x)}+\text { h.c. }\right]
$$

where we have rewritten (1.9) and (1.11) as an action. We have reintroduced $\hbar$ and other pesky constants to show explicitly the various physical limits. Note that although we are mainly concerned here with fermions (1.9) describes in fact nearly every one dimensional disordered problem ranging from dirty bosons 19, 20, 21 to disordered spin chains 22], since all this problems have essentially the same boson representation. We will examine these different systems in more details in section 4 (1.13) emphasizes the physics of the problem. The electron system can be viewed as a charge density wave 23, 24. since the density varies as (1.6)

$$
\rho(x, \tau)=\rho_{0} \cos \left(2 k_{F} x-2 \phi(x, \tau)\right)
$$

The elastic term in (1.13) wants the phase of the density wave to be as constant as possible, and have a nice sinusoidally modulated density. The disorder term on the contrary wants to pin this charge density on the impurities by distorting the phase, as shown on Figure 1. The problem of localization of interacting fermions is thus very similar to the one of the pinning of classical charge density waves 25. The charge density wave is

here intrinsic to the one-dimensional interacting electron gas and not due 


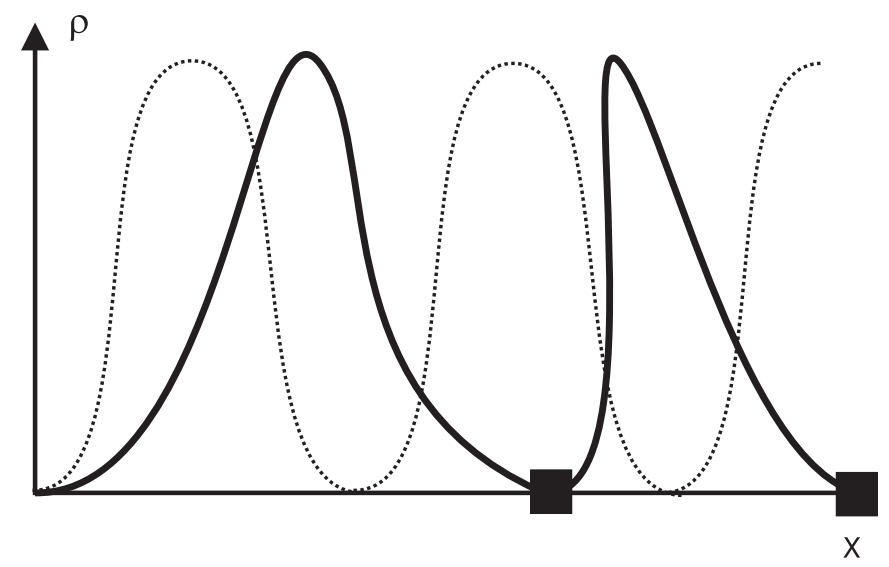

FIGURE 1. Impurities (black square) will pin the density wave. The phase $\phi$ will have to change compared to the undistorted density (dotted line), which costs kinetic energy.

to a coupling to phonons. The main features are nevertheless similar, the main difference being the fact that the effective mass of the "CDW" is much smaller in the absence of the electron-phonon coupling and hence the importance of the quantum fluctuations is much higher. Various quantities of special interest have a quite simple expression in terms of the bosons. The conductivity is simply given by

$$
\sigma(\omega)=\frac{i}{\omega}\left[\left\langle\partial_{\tau} \phi \partial_{\tau} \phi\right\rangle_{i \omega_{n}, q=0}\right]_{i \omega_{n} \rightarrow \omega+i \delta}
$$

whereas the compressibility is given by a similar correlation function but with differents limits

$$
\kappa=\frac{1}{\pi^{2}}\left\langle\partial_{x} \phi \partial_{x} \phi\right\rangle_{i \omega_{n}=0, q}
$$

The correlations of the $2 k_{F}$ part of the density and of the superconducting order parameter $\psi_{+}^{\dagger} \psi_{-}^{\dagger}$ are respectively given by

$$
\begin{aligned}
& \chi_{\rho}(r)=\left\langle e^{i 2 \phi(r)} e^{-i 2 \phi(0)}\right\rangle \\
& \chi_{s}(r)=\left\langle e^{i 2 \theta(r)} e^{-i 2 \theta(0)}\right\rangle
\end{aligned}
$$

where $r=(x, \tau)$ and $\pi \Pi(x)=\nabla \theta(x)$.

Given the highly nonlinear nature of the coupling to disorder, (1.13) is quite tough to solve. On the other hand, if $\xi(x)$ was just a constant we would have a simple sine-Gordon theory for which a great deal is known. We will thus tackle the problem by increasingly sophisticated methods. 


\subsection{Chisel and Hammer}

In the absence of quantum fluctuations, $\phi$ would be a classical field and we would have a good idea of what happens. This is the way Fukuyama and Lee 25 looked at this problem. Such an approximation is of course very good for "phononic" charge density waves 23 since the quantum term is $\Pi^{2} /(2 M)$ and thus very small. For fermions this corresponds to the "classical" limit $\hbar \rightarrow 0, K \rightarrow 0$ keeping $\bar{K}=K / \hbar$ fixed, and thus to very repulsive interactions. In that case we can ignore all quantum fluctuations, and look for a static solution for $\phi$. It is of course crucial for the existence of such solution that the disorder does not depends on time. This solution $\phi_{0}(x)$ describes the static distortion of the phase imposed by the random potential. In the absence of kinetic energy $(\nabla \phi)^{2}$, it would be easy to "determine" $\phi_{0}$. If we write the random field $\xi$ as an amplitude $|\xi(x)|$ and a random phase $2 \zeta$, the disorder term writes

$$
\int d x|\xi(x)| e^{i 2(\phi(x)-\zeta(x))}+\text { c.c. }
$$

The optimum is thus for $\phi_{0}(x)$ to follow the random phase on each point. For point like impurities located on random positions $R_{i},|\xi|$ would just be the strength of each impurity potential and $\zeta=k_{F} R_{i}$. Thus $\phi_{0}(x)=\zeta(x)$ is the generalisation to any type of disorder (and in particular to the Gaussian disorder so dear to the theorist) of the physics expressed in Figure 1: get the density minimum at each impurity. In presence of kinetic energy following the random phase would cost too much kinetic energy. We do not know exactly now how to determine the optimal $\phi_{0}(x)$ but we can do some scaling arguments. Let us assume that $\phi$ remains constant for a lengthscale $L_{\text {loc }}$. On this lengthscale $\phi$ takes the value that optimizes the disorder term, which now reads

$$
E_{\mathrm{dis}}=\left[\int_{0}^{L_{\mathrm{loc}}} \xi(x)\right] e^{i 2 \phi}+\text { c.c. }
$$

If we take for example a gaussian distribution for $\xi$

$$
\overline{\xi(x) \xi^{*}\left(x^{\prime}\right)}=\mathcal{D} \delta\left(x-x^{\prime}\right)
$$

one gets because of the average of a complex random variable on a box of size $L_{\text {loc }}$ that the disorder contributes as

$$
E_{\mathrm{dis}}=-\sqrt{\mathcal{D} L_{\mathrm{loc}}} e^{i\left(2 \phi_{0}-2 \Xi\right)}
$$

where $\Xi$ is some phase. It clear that the optimum energy is reached if $\phi_{0}$ adjusts to this (now unknown) phase. The global energy gain now scales as $\sqrt{L_{\text {loc }}}$. Between two segments of size $L_{\text {loc }}$ the phase has to distort to reach the next optimal value. The distortion being of the order of $2 \pi$ the cost in kinetic energy reads

$$
E_{\text {kin }} \propto \frac{1}{L_{\text {loc }}}
$$


minimizing the total cost shows that the length over which $\phi_{0}$ remains constant is given by

$$
L_{\mathrm{loc}} \propto\left(\frac{1}{\mathcal{D}}\right)^{\frac{1}{3}}
$$

This tells us that the system does pin on the impurities and that below $L_{\mathrm{loc}}$ the system looks very much like an undistorted system. Since at the scale $L_{\mathrm{loc}}, \phi_{0}$ varies randomly the $2 k_{F}$ density density correlations will decay exponentially with a characteristic size $L_{\text {loc }}$. It is thus very tempting to associate $L_{\text {loc }}$ with the Anderson localization length. Note that for the free fermion point $L_{\text {loc }} \propto 1 / \mathcal{D}$ instead of (1.24), so the above formula is clearly missing a piece of physics when $K$ is not zero. Nevertheless from this simple scaling argument we have obtained: (i) the fact that classical CDW or very very repulsive fermions are pinned (localized) by disorder; (ii) the localization length; (iii) the fact that the ground state should contain a static distortion of the phase due to the disorder. Unfortunately we have no other information on $\phi_{0}$, which is certainly a drawback.

Even with our limited knowledge of the statics we can nevertheless try to extract the dynamics. Let us assume that all deformations of the phase which are not contained in the static distortion are small and thus that we can write

$$
\phi(x, \tau)=\phi_{0}(x)+\delta \phi(x, \tau)
$$

with $\delta \phi(x, \tau) \ll \phi_{0}(x)$ in a very vague sense since we deal with random variables. One can try to expand the random term in power of $\delta \phi$

$$
\begin{aligned}
S_{\mathrm{dis}} & =\int d \tau d x|\xi(x)| \cos (2(\phi(x, \tau)-\zeta(x))) \\
& \simeq-2 \int d \tau d x|\xi(x)| \cos \left(2\left(\phi_{0}(x)-\zeta(x)\right)\right)(\delta \phi(x, \tau))^{2}
\end{aligned}
$$

One can thus use in principle (1.26) to compute the various physical quantities. Note that the conductivity 1.15) will not depend directly on the statics solution $\phi_{0}$ since $\partial_{t} \phi_{0}=0$, so we can hope to compute it. Of course the dependence of the fluctuations $\delta \phi$ in $\phi_{0}$ is hidden in $(1.26)$. If $\phi_{0}$ was following the random phase at every point, then the disorder term would just lead to a mass term for the fluctations and the optical conductivity would show a gap. In fact this is not true at every point so $(1.26)$ leads to a distribution of masses for the fluctuations. Unfortunately the knowledge of $\phi_{0}$ is too crude to compute the conductivity accurately and depending on what exactly is $L_{\mathrm{loc}}$ one can find either a gap, a non analytic behavior or a $\sigma(\omega) \sim \omega^{2}$ behavior at small frequencies 25. Based on physical intuition Fukuyama and Lee opted for the later 25], but the method shows its limitations here and does not allow a reliable calculation of the physical quantities. More precise calculations of $\phi_{0}$ and the conductivity can be performed in the classical limit $K \rightarrow 0$ using a transfer matrix formalism [26]. 
One thing that can be obtained from the partial knowledge of the fluctuations is the effect of quantum fluctuations 27]. Indeed when quantum fluctuations are present the expansion (1.26) is not valid any more since the cosine should be normal ordered before it can be expanded. This leads to

$$
\cos \left(2\left(\phi_{0}-\zeta+\delta \phi\right)\right) \simeq-2 \cos \left(2\left(\phi_{0}-\zeta\right)\right) e^{-2\left\langle\delta \phi^{2}\right\rangle}(\delta \phi)^{2}
$$

where the average \langle\rangle has now to be computed self-consistently using (1.27). This leads to a modified localisation length 27] of the form

$$
L_{\mathrm{loc}} \propto\left(\frac{1}{\mathcal{D}}\right)^{\frac{1}{3-2 K}}
$$

This expression for the localization length suggests that a delocalization transition is induced by the quantum fluctuations and occurs at $K=3 / 2$. In the fermion language this corresponds to extremely attractive interactions.

\subsection{Starting from the metal: $R G$}

The previous method starts directly from the localized phase. It provides some limited information about this phase, but suffers from serious limitations. An alternative approach is to start from the pure Luttinger liquid and investigate the effects of disorder perturbatively, and build a renormalization group analysis. The RG provides us with the best possible description of the delocalized phase and the critical properties of the transition. It also gives a very accurate description of the localized phase up to lengthscales of the order of the localization length $L_{\text {loc }}$. Here again we describe the method for simplicity on spinless fermions and discuss more complex systems in section 4 .

To have a hint of the RG equations let us expand the disorder term (1.13) to second order. This leads to

$$
\int d \tau d x \int d \tau^{\prime} d x^{\prime} \xi(x) \xi^{*}\left(x^{\prime}\right) e^{2 i\left(\phi(r)-\phi\left(r^{\prime}\right)\right)}
$$

It is easy to see that at the tree level, (1.29) scales as $\mathcal{D} L^{3-2 K}$. This leads to the scaling of the disorder

$$
\frac{\partial \mathcal{D}}{d l}=(3-2 K) \mathcal{D}
$$

This traduces in fact the dressing of the scattering on the disorder by the interactions and has been derived using either diagrams or RG [28, 29, 30, 31, 32, 33, 19, 20,. In itself it seems to confirm the result of (1.28) i.e. the existence of a transition. Note that the advantage of the bosonization derivation is to allow to reach the non perturbative point in interactions where such a metal-insulator transition would take place. 
In fact (1.30) would not allow in itself to really determine the metalinsulator transition point. This can be seen by using the RG to compute the finite temperature (or finite frequency) conductivity of the system [19, 20]. The idea is simply to renormalize until the cutoff is of the order of the thermal length $l_{T} \sim u / T$ corresponding to $e^{l^{*}} \sim l_{T} / \alpha$. At this length scale the disorder can be treated in the Born approximation. As the conductivity is a physical quantity it is not changed under renormalization and we have:

$$
\sigma(n(0), D(0), 0)=\sigma(n(l), D(l), l)=\sigma_{0} \frac{n(l) D(0)}{n(0) D(l)}=\sigma_{0} \frac{e^{l} D(0)}{D(l)}
$$

where $\sigma(n(l), D(l), l)=\sigma(l)$ and $n(l)$ are respectively the conductivity and the electronic density at the scale $l . \sigma_{0}=e^{2} v_{F}^{2} / 2 \pi \hbar \mathcal{D}$ is the conductivity in the Born approximation, expressed with the initial parameters. Using (1.30) one gets from (1.31)

$$
\sigma(T) \sim \frac{1}{\mathcal{D}} T^{2-2 K}
$$

This result is the direct consequence of the renormalization of the scattering on impurities by interactions (1.30). One immediately sees that (1.32) alone would lead to a paradox since (1.30) gives a localized-delocalized boundary at $K=3 / 2$ whereas (1.32) gives perfect conductivity above $K=1$ (i.e. the noninteracting point). One could also immediately see that if one introduces a new variable such as

$$
\tilde{\mathcal{D}}=e^{-a l} \mathcal{D}
$$

the dimension of such a variable would be $(3-a-2 K)$, leaving the location of the transition point as determined from (1.30) quite arbitrary. Although such a transformation seems arbitrary, if one considers that the disorder stems from impurities with a concentration $n_{i}$ and a strength $V$, the limit of Gaussian disorder corresponds simply in taking $n_{i} \rightarrow \infty$ with $V \rightarrow 0$ keeping $\mathcal{D}=n_{i} V^{2}$ fixed. Thus the choice $a=1$ in (1.33) simply corresponds to $\tilde{\mathcal{D}}=V^{2}$ i.e. writting an RG equation for the impurity strength.

The answer to this simple paradox is of course that (1.30) should be complemented by another RG equation. In addition to renormalizing $\mathcal{D}$ (1.29) generates as well quadratic terms that renormalize the free part of the Hamiltonian, i.e. the velocity $v$ and the Luttinger parameter $K$. Details can be found in 19,20 . The main equation is the renormalization of the Luttinger parameter $K$ and reads

$$
\frac{\partial K}{d l}=-K^{2} \mathcal{D} / 2
$$

This equation describes the renormalization of the interactions by the disorder. Both RG equations (1.30) and (1.34) have a diagramatic representation shown on figure 2. Using the flow (1.30) and (1.34) one can easily check 


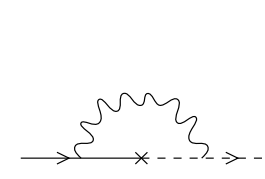

a) 1

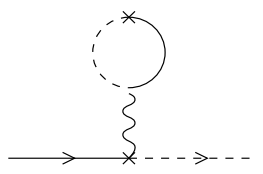

a) 2

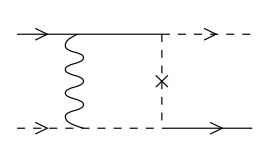

b)

FIGURE 2. Diagrams describing the renormalization of the disorder by the interactions (a) and the renormalization of the interactions by the disorder (b). Solid and dotted lines are fermions with $\pm k_{F}$, the wiggly line is the interaction and the cross is the impurity scattering.

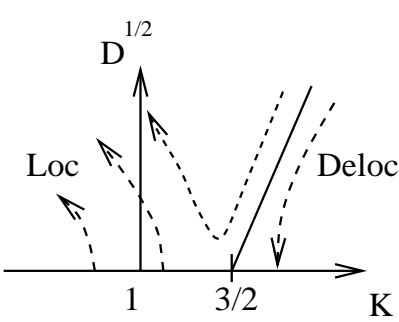

(a)

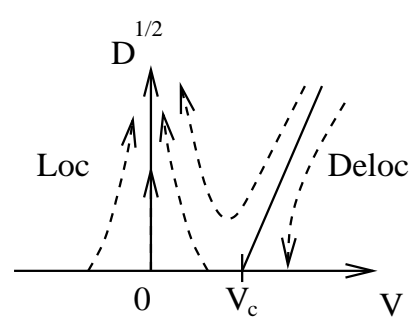

(b)

FIGURE 3. Phase diagram and flow for spinless fermions in presence of disorder. (a) is the flow in $\mathcal{D}$ and $K$ variables. (b) the flow in the $\mathcal{D}$ and interactions $g$. Disorder kills inelastic interactions. 
that two phases exist as shown on figure 3. For large $K$ one in the delocalized phase where the disorder is irrelevant and the system is a Luttinger liquid with renormalized coefficients $u^{*}$ and $K^{*}$. All correlation functions decay as power laws, and because $K^{*}>3 / 2$ the system is dominated by superconducting fluctuations. On the transition line the exponent flows to the universal value $K^{*}=3 / 2$ Below this line $\mathcal{D}$ flows to large values, indicating that the disorder is relevant. This phase is the localized phase. This is obvious for physical reasons but can also be guessed from the exact solution known for the noninteracting line $K=1$ (and any $\mathcal{D}$ ) which belongs to this phase. As can be seen from $(1.30)$ and $(1.34)$ the transition is Berezinskii-Kosterlitz-Thouless (BKT) like 34, 35 in the $K, \sqrt{\mathcal{D}}$ variables.

In addition to the phase diagram itself a host of physical properties can be extracted from the RG. The simplest one is the localization length. One can use that for $\mathcal{D}(l) \sim 1$ the localization length is of the order of the (renormalized) lattice spacing $\alpha e^{l}$. The full determination needs an integration of both (1.30) and (1.34). Close to the transition the divergence of the localization length is BKT like (setting $K=3 / 2+\eta$ )

$$
L_{\mathrm{loc}} \sim e^{2 \pi / \sqrt{9 \mathcal{D}-\eta^{2}}}
$$

Deep in the localized regime, and for weak disorder, a good approximation is to neglect the renormalization of $K$ in (1.30). A trivial integration of (1.30) then gives back (1.28). This we see that the SCHA calculation corresponds in fact, both for the phase diagram and for the localization length to the limit of infinitesimal disorder.

Out of the RG one can also extract, using (1.31) the behavior of the temperature or frequency dependence of the conductivity. In the localized phase this can only be used up to the energy scale corresponding to the localization length i.e. $E_{\text {pin }}=k_{B} T_{\text {pin }}=\hbar \omega_{\text {pin }}, \omega_{\text {pin }}=v / L_{\text {loc }}$. Below this lengthscale another method than the weak coupling RG should be used. We will come back to that point in section 6. Here again, although the full flow should be taken into account one can get an approximate formula by ignoring the renormalization of $K$, which leads to (1.32). For $K<3 / 2$ (including the noninteracting point) any small but finite disorder grows, renormalizing the exponents and ultimately leading to a decrease of the conductivity, even if one started initially from $K>1$. A very crude way of taking into account both equations $(1.30)$ and $(1.34)$ would be to say that one can still use (1.32) but with scale dependent exponents (see [19, 20])

$$
\sigma(T) \sim T^{2-2 K(T)}
$$

This renormalization of exponents and the faster decay of conductivity is in fact the signature of Anderson localization. The equivalent frequency dependence is

$$
\sigma(\omega) \propto \omega^{2 K-4}
$$


Here again this formula break down below the scale $\omega_{\text {pin }} \sim v / L_{\mathrm{loc}}$ which is the pinning frequency. Similarly below $L_{\text {loc }}$ correlation functions can again be computed using the RG, but of course the asymptotic behavior cannot be obtained.

\section{Other systems and RG}

Despite its limitations to physics above $E_{\text {pin }}$ the RG is an extremely efficient method given its simplicity. It allows in addition a perfect description of the delocalized phase and of the critical behavior, something unatainable through the methods of section 3.1 and allows for interesting extensions.

First let us note that the equations $(1.30)$ and $(1.34)$ also describe the case of a single impurity 36, 37. Indeed in that case one can go back to the definition $\mathcal{D}=n_{i} V^{2}$ and take the limit $n_{i} \rightarrow 0$, i.e. $\mathcal{D} \rightarrow 0$. (1.34) shows that in that case $K$ cannot be renormalized since a single impurity cannot change the thermodynamic behavior. Only (1.30) remains, leading directly to temperature dependence of the form (1.32), and a localizeddelocalized transition at $K=1$. More details on such a relation between the two problems and on the remaining open question can be found in [38].

Quite remarkably the set $(1.30-1.34)$ seems wrong. Indeed $K$ naively depends on the (inelastic) interations. Perturbatively, for the pure systems $K=1-V /\left(2 \pi v_{F}\right)$. If one start for $K=1$, i.e. for the non interacting system, it would thus seem from (1.34) that the elastic scattering on the impurities can generate inelastic fermion-fermion interactions. The solution of this paradox is hidden in the precise way the RG procedure is build. In order to have the elastic nature of the scattering on impurities, the time integrations in (1.29) should be dome independently for $\tau$ and $\tau^{\prime}$. When one performs the RG one introduces a cutoff and imposes $\left|\tau-\tau^{\prime}\right|>\alpha$. Thus a part is left out of (1.29) which is

$$
\begin{aligned}
\mathcal{D} \int & d x \int_{\left|\tau-\tau^{\prime}\right|<\alpha} d \tau d \tau^{\prime} \rho(x, \tau) \rho\left(x, \tau^{\prime}\right) \\
& \simeq 2 \mathcal{D} \alpha \int d x \int d \tau \rho(x, \tau) \rho(x, \tau)
\end{aligned}
$$

which is exactly an inelastic interaction term. Thus in fact $K$ contains not only the original inelastic interactions $V$ but also a small correction coming from the disorder itself. In order to determine the flow for $V$ it is thus necessary to take this small correction into account [19, 20] which gives the flow of Figure $3-b$. One thus sees that the elastic case $V=0$ indeed remains elastic and also that for spinless fermions, the perturbative flow seems to indicate that the inelastic interactions are reduced by the disorder. This is compatible with the physical image that one would get at strong disorder: 
fermions localize individually and since the overlap of wavefunctions is exponentially small, so is the effect of interactions. One could thus naively expect that below $L_{\text {loc }}$ the effect of interactions are strong but disappear above $L_{\mathrm{loc}}$. As we will see in section 6 the variational approach confirms this image. For fermions with spins (to be discussed below) we expect only the interaction in the charge sector to vanish. Interactions in the spin sector do remain and lead to random exchange.

Of course many more physical systems can be studied by this method. This is the case for spin chains, than can directly be mapped onto spinless fermions 18. A spin chain under a random magnetic field along $z$ 222 is directly the problem that we solved in the previous section, with $K$ being the anisotropy ( $K=1$ for an XY chain and $K=1 / 2$ for an Heisenberg one). However although for fermion problems it is unlikely that one is at a commensurate filling this is the natural situation for a spin chain (since the magnetization is zero in the absence of external field the filling of the equivalent spinless fermion system is $n=1 / 2$ ). A spin chain with random exchange is thus like a commensurate fermionic system with random hopping. We will come back to this peculiar case in section 7 . Quite remarkably, in one dimension, bosons 19, 20 lead to physics similar to fermions. This is due to the fact that in one dimension statistics cannot be separated from interactions. Interacting bosons can thus be represented by a bosonization representation quite similar to the one of fermions 39. The density can be written as

$$
\rho(r)=\rho_{0}-\rho_{0} \partial_{x} \phi+\sum_{n} e^{i n\left(2 \pi \rho_{0} x-2 \phi(r)\right)}
$$

very similar to the fermionic form. while the single particle operator is now

$$
\psi(r) \sim \rho_{0}^{1 / 2} e^{i \theta}
$$

(note the difference with the fermionic operator). The hamiltonian is still described by the quadractic form (1.13). Now $K=\infty$ for noninteracting bosons and $K=1$ for hard core ones. There is thus a transition 19, 20 between a superfluid state (for $K>3 / 2$ ) to a Bose glass state where the bosons are localized by the random potential at $K=3 / 2$.

In a similar way one can of course treat fermions with spins. Equations are more complicated since they involve the charge and spin sectors, and we will not discuss the full physics here but refer the reader to [20]. Let us just draw attention to one interesting consequence of the renormalization equation of the disorder for the problem with spins, which reads

$$
\frac{d \mathcal{D}}{d l}=\left(3-K_{\rho}-K_{\sigma}-g_{1 \perp}\right) \mathcal{D}
$$

where $g_{1 \perp}$ is the backscattering interaction between opposite spins. For a Hubbard type interaction $g_{1 \perp}=U$. For spin isotropic systems $K_{\sigma}=$ 
$1+g_{1 \perp} /\left(2 \pi v_{F}\right)$ and $g_{1 \perp}$ is marginal with a flow

$$
\frac{d g_{1 \perp}}{d l}=-g_{1 \perp}^{2}
$$

For more general spin couplings either $g_{1 \perp} \rightarrow 0$ and $K_{\sigma} \rightarrow K_{\sigma}^{*}$, or $g_{1 \perp}$ is relevant and a spin gap opens. The physics of the localization transition depends thus on the sign of the interactions with special physical consequences. The transition point move from $K_{\rho}=2$ (for infinitesimal disorder) for repulsive interactions to $K_{\rho}=3$ for attractive ones. For a Hubbard type interaction for which $1 / 2<K_{\rho}<2$ the delocalization point can never be reached and the system is localized regardless of the strength of interaction and disorder. Another consequence is that in the presence of spin degrees of freedom the divergence of the localization length at the transition is not BKT like any more. One could naively think that in the repulsive case the $g_{1 \perp}$ term could be omitted and that the renormalization of the disorder could be written $\left(3-K_{\rho}-K_{\sigma}^{*}\right)$. For spin isotropic interactions this misses an important part of the physics. Indeed, for a Hubbard interaction $K_{\rho}=1-U /\left(2 \pi v_{F}\right)$. Substituting in (1.41) leads (for the initial steps of the flow)

$$
\frac{d \mathcal{D}}{d l}=\left(1-\frac{U}{\pi v_{F}}\right) \mathcal{D}
$$

whereas the incorrect substitution at the fixed point would lead to $\left(1+\frac{U}{\pi v_{F}}\right)$, leading to quite different physics. (1.43) implies that for Hubbard type interactions repulsive interactions make the system less localized th0 than for attractive interactions, i.e. that the $L_{\mathrm{loc}}^{U>0}>L_{\mathrm{loc}}^{U<0}$. Similar effects exist for the charge stiffness and the persistent currents, i.e. for a system with spins the persistent currents are in fact enhanced by repulsive interactions. This counter-intuitive statement can be explained physically: interactions have two effects: (i) they tend to reinforce, when attractive the superconducting fluctuations in the system. This screens disorder and makes it less effective. This is the only effect occurring for spinless fermions. (ii) when spin degrees of freedom exists, repulsive interactions also tend to make the density more uniform by spreading the charge. This makes it more difficult to couple to disorder. These two effect compete. This has several consequences, in particular for mesoscopic systems. Of course, for fermions, true delocalization can only be achieved with attractive interactions reaching at least the nearest neighbor.

Many other systems have been treated by such method. there are onedimensional systems with long-range $1 / r$ interactions, that lead to a pinned Wigner crystal [41], doped spin 1 chains [42], fermionic [43] and bosonic [44] ladders, spin 1 chains in a magnetic disorder [45], spin ladders 446]. Since we want to focus here on the methods we refer the reader to the above references for a detailed discussion of the physics of such systems. 


\section{A zest of numerics}

Although we are mainly concerned about analytical method in these notes, let us mention some numerical results and methods that have been used in connection with the RG predictions. Although numerical studies have become very powerful in one dimension for pure systems the presence of disorder complicates matters. Three main methods have been used.

Exact diagonalizations, have been used to study both the phase diagram and the charge stiffness of both spinless fermions 47, 48] (or equivalently XXZ spin chains) and fermions with spins 49,50 with short range or long range interactions 51]. Using the finite size scaling of the spin stiffness $\rho_{s}=\frac{1}{L} \frac{\partial^{2} E(\theta)}{\partial \varphi^{2}}=f\left(\frac{L}{L_{\text {loc }}}\right)$, where $E(\theta)$ is the ground state energy of the disordered XXZ chain with boundary conditions $S_{L}^{+}=e^{i \varphi} S_{1}^{+}$, the localization length $L_{\text {loc }}$ can be obtained [47], and is in good agreement with the RG results of section 3.2. The behavior of the correlation length close to the transition point appeared consistent with the predicted BKT-like behavior. The results also suggested that a finite disorder was needed to disorder the ground state for $K>3 / 2$. A similar study with systems sizes of up to $L=18$ sites was also made for XXZ spin chains with a random exchange in [52. Analysis of persistent currents was also in agrement with the RG prediction of section 1 . Unfortunately the exact Diagonalization approach of the last section is limited to zero temperature and small system size.

In order to consider bigger system sizes, one can use Quantum Monte Carlo methods. In [53], such a study was performed for disordered bosons. The superfluid density was obtained as a function of interaction for a given disorder strength. It was shown that for not too repulsive equations, there was a phase with a finite superfluid density. For more repulsive interactions, a phase with finite compressiblity by zero superfluid density was obtained, in agreement with the Bose Glass theory of section 1 .

The most promising recent method is the Density Matrix Renormalization Group. It been introduced in the recent years as a method specially designed to calculate the ground state of correlated one-dimensional systems 54. This method has been also applied to the problem of the XXZ chain in a random magnetic field parallel to the $\mathrm{z}$ axis by Schmitteckert et al. [55. The authors of [55] have been able to consider system size of up to $L=60$ sites, and average over several hundred realizations of the disorder. Localization and phase diagram were also in good agreeement with the RG predictions.

Clearly, various numerical checks confirm the predictions of the RG. Unfortunately so far only the phase diagram, stiffness and localization length have been computed. This is clearly related to the complexity of the problem at hand. What would be extremely useful would be informations on quantities deep in the localized phase such as the single particle Green's function, the ac or dc conductivity. Analysis of such quantities would nicely 
complement the RG analytical study and allow for comparison with other analytical techniques more suited for the localized phase such as the variational method we analyze in the next section.

\section{Variational Method}

Let us now study this problem using a completely different and at first sight more formal method. As usual it is very convenient to get rid of the disorder from the start. Given the non quadratic nature of (1.13) supersymmetric methods are unapplicable and we have to turn to replicas. The idea of the replica method in itself is quite simple. If we want to compute an observable $O$ we have to do both average over disorder and thermodynamic average

$$
\overline{\langle O\rangle}=\int \mathcal{D} V p(V)\langle O\rangle_{V}=\int D V p(V) \frac{\int \mathcal{D} \phi O[\phi] e^{-S_{V}[\phi]}}{\int D \phi e^{-S_{V}[\phi]}}
$$

The action is usually linear in disorder and for Gaussian disorder the distribution of random potential is $p(V) \propto e^{-\int d x V(x)^{2}}$, so the average would be quite trivial without the denominator in (1.44). The idea is thus to introduce $n$ fields and to compute

$$
\begin{gathered}
\int D \phi_{1} D \phi_{2} \ldots D \phi_{n} O\left[\phi_{1}\right] e^{-\sum_{i=1}^{n} S_{V}\left[\phi_{i}\right]}= \\
\int D \phi O[\phi] e^{-S_{V}[\phi]}\left[\int D \phi e^{-S_{V}[\phi]}\right]^{n-1}
\end{gathered}
$$

which is exactly the quantity we want to average over disorder in (1.44) if one takes the formal limit $n \rightarrow 0$. Since (1.45) has no denominator averaging over disorder is trivial. Of course there is a price to pay: before the averaging the replicas are all independent fields but the averaging introduces an interaction between them. We have thus traded a theory depending on a random variable $V$ but a single field for a theory without disorder but with $n$ coupled fields. Usually this is still a situation we are better equipped to solve because of the large number of field theoretic method dealing with "normal" (i.e. transitionally invariant actions). For the particular case (1.13) the replicated action is

$$
\begin{aligned}
S / \hbar= & \int d x d \tau \frac{1}{2 \pi K} \sum_{a}\left[\frac{1}{v}\left(\partial_{\tau} \phi_{a}\right)^{2}+v\left(\partial_{x} \phi_{a}\right)^{2}\right]- \\
& \frac{\mathcal{D}}{(2 \pi \alpha)^{2} \hbar} \sum_{a b} \int d x d \tau d \tau^{\prime} \cos \left(\phi_{a}(x, \tau)-\phi_{b}\left(x, \tau^{\prime}\right)\right)
\end{aligned}
$$

where $a=1, \ldots, n$ is the replica index. Disorder averaging has coupled the replicas via the cosine term. Because the disorder is time independent 
this coupling contains two fields that can be at arbitrary time and is thus highly non local. For fermions one usually prefers to go to frequency space, where this imply conservation of the frequency for each replica index, but this would not simplify things here because of the cosine.

This is up to now a totally formal procedure and nothing has been accomplished. (1.46) is totally equivalent to $(1.13)$ and the difficulty is of course to solve it. Based on the RG equation (1.30) one could think naively that since the localized phase corresponds to $\mathcal{D} \rightarrow \infty$ it would be safe to expand the cosine term in (1.46). Unfortunately it is easy to check that fails seriously when $n \rightarrow 0$ is taken (it of course works perfectly for a finite number of field $n \geq 2$ ). In order to circumvent this problem let us try to improve over this simple minded expansion of the cosine. Let us try a variational ansatz. We introduce a trial action $S_{0}$

$$
S_{0} / \hbar=\frac{1}{2 \beta L \hbar} \sum_{q, \omega_{n}} \sum_{a b} \phi_{a}\left(q, \omega_{n}\right) G_{a b}^{-1}\left(q, \omega_{n}\right) \phi_{b}\left(-q,-\omega_{n}\right)
$$

where the propagators $G^{-1}$ are our variational "parameters". As usual $\frac{1}{L} \sum_{q} \rightarrow \int \frac{d q}{(2 \pi)}$. If we introduce

$$
Z=\int \mathcal{D} \phi e^{-S / \hbar}
$$

We then have the variational theorem for the free energy $F=-\hbar \log (Z)$

$$
F \leq F_{\mathrm{tr}}=F_{0}+\left\langle S-S_{0}\right\rangle_{S_{0}}
$$

Since $S_{0}$ is quadratic, $(1.50)$ can be in general computed quite explicitely as a function of the (unknown) propagators $G$. The "best" quadratic action $S_{0}$ is thus the one that satisfies the saddle point equations

$$
\frac{\partial F_{\mathrm{tr}}}{\partial G_{a b}\left(q, \omega_{n}\right)}=0
$$

which gives a set of integral equations allowing to determine the unknown functions $G$.

The observables are simply defined by quantities diagonal in replica indices as can be seen from (1.45). For some quantities such as the compressibility it is necessary to be more careful since one has to substract the average, which is usually zero in a pure system or after averaging over disorder but non zero for a specific realization of the disorder. Let us introduce the various propagators (time ordering in $\tau$ is always implied):

$$
\begin{aligned}
B_{a b}(x, \tau)= & \left\langle\left[\phi_{a}(x, \tau)-\phi_{b}(0,0)\right]^{2}\right\rangle= \\
& \left(G_{a a}(0,0)+G_{b b}(0,0)-2 G_{a b}(x, \tau)\right) \\
G_{a b}\left(q_{x}, \omega_{n}\right)= & \left\langle\phi_{a}\left(q_{x}, \omega_{n}\right) \phi_{b}\left(-q_{x},-\omega_{n}\right)\right\rangle
\end{aligned}
$$


The compressibility is given by

$$
\begin{aligned}
\chi\left(q, \omega_{n}\right)= & \frac{1}{\hbar} \int d x \int_{0}^{\beta \hbar} d \tau e^{-\imath\left(q x-\omega_{n} \tau\right)} \times \\
& \times \frac{\left\langle T_{\tau}(n(x, \tau)-\langle n(x, \tau)\rangle)(n(0,0)-\langle n(0,0)\rangle)\right\rangle}{1,1,}
\end{aligned}
$$

which leads to the average static compressibility $\chi_{s}=\lim _{q \rightarrow 0}\left(\lim _{\omega \rightarrow 0} \chi(q, \omega)\right)$ (see $(1.16))$. When expressed in terms of the replicated bosonized operators (1.54) gives

$$
\chi_{s}=\lim _{q \rightarrow 0} \lim _{\omega \rightarrow 0} q^{2} G_{c}(q, \omega)
$$

where we introduced an important propagator: the connected one defined as $G_{c}^{-1}(q)=\sum_{b} G_{a b}^{-1}(q)$.

Without the replicas this method is nothing but the well known Self Consistent Harmonic Approximation (SCHA), which is known to work very well for sine-Gordon type Hamiltonians. Such a method gives in particular correctly the two phases (massless and massive). Extension of this method to disordered systems was done in the context of classical elastic systems such as interfaces [56]. In quantum problems another level of complexity occurs because of the aforementioned non locality of the interaction in time. But before going to these problems, specific to the quantum systems, let us illustrate the aspects of this variational method when applied to disordered systems, on a technically simpler example (for which this method was extremely fruitful 57): the case of classical periodic systems.

\subsection{A classical example}

Let us take the action (1.46) but with only a single time integral for the disorder term. Such action would be the result of the average on a disorder both dependent on space and time. Of course such a disorder would be quite unrealistic for quantum problems. However (1.56) would be a perfectly natural Hamiltonian for a classical problem where $z=v \tau$ is now just one of the spatial dimensions [58, 57. To make the analogy more transparent let us use $z=v \tau$, and replace the integral over $x$ by an integral in $d-1$ dimensions. If denote by $r$ the $d$-dimensional space variable $r=(x, z)$ the starting action is

$$
\begin{aligned}
S / \hbar & =\int d^{d} r \frac{1}{2 \pi K} \sum_{a}\left(\partial_{r} \phi_{a}\right)^{2} \\
& -\frac{\mathcal{D}}{(2 \pi \alpha)^{2} \hbar v} \sum_{a b} \int d^{d} r \cos \left(2\left(\phi_{a}(r)-\phi_{b}(r)\right)\right)
\end{aligned}
$$

One can see that 1.56) is exactly the Hamiltonian describing an elastic system such as a vortex lattice or a classical CDW in the presence of point like defects in $d$ dimensions. $\hbar$ plays the role of the temperature for the 
classical system, the elastic constant $c$ is given by $c=1 /(\pi \bar{K})$ with $\bar{K}=$ $K / \hbar$ and $\rho_{0}^{2} \Delta / 2=\mathcal{D} \hbar /(2 \pi \alpha)^{2}$ would be the correlator of the classical disorder 58 .

If we call $q=\left(q_{x}, \omega\right)$ the $d$-dimensional momentum, without loss of generality, the matrix $G_{a b}^{-1}(q)$ can be chosen of the form $G_{a b}^{-1}=c q^{2} \delta_{a b}-\sigma_{a b}$. We obtain by minimization of the variational free energy the saddle point equations

$$
G_{c}^{-1}(q)=c q^{2}, \quad \sigma_{a \neq b}=\frac{2 \mathcal{D}}{(\pi \alpha)^{2}} e^{-2 B_{a b}(r=0)}
$$

Using $(1.52-1.53)$ one obtains

$$
B_{a b}(r)=\hbar \int \frac{d^{d} q}{(2 \pi)^{d}}\left(G_{a a}(q)+G_{b b}(q)-2 \cos (q r) G_{a b}(q)\right)
$$

For this particular problem, the connected part is not affected by disorder. This is the consequence of a hidden symmetry (statistical tilt symmetry) of (1.56), whose disorder part is not affected by any local shift of $\phi_{a}(r)$ such as $\phi_{a}(r) \rightarrow \phi_{a}(r)+f(r)$, where $f$ is an arbitrary function. Such a symmetry does not exist for the time correlated disorder natural in a quantum problem, with important physical consequences on which we will come back. The only interesting equation here is thus the equation for the off-diagonal part $\sigma_{a \neq b}$. Because of the locality of the interaction term between replicas in (1.56) the self energy $\sigma_{a b}$ is simply a matrix of constants.

Given the symmetry of the original action/Hamiltonian (1.56) by permutation of the replica indices, it is very natural to look for a variational ansatz with the same symmetry. This would mean that the $G^{-1}$ matrix would have only (for each value of $q$ ) two independent values: the diagonal one $G_{a a}=\tilde{G}$ and the off diagonal one $G_{a \neq b}$. Such a matrix can easily be inverted for any $n$, and the analytic continuation for $n \rightarrow 0$ gives

$$
\begin{aligned}
G_{c} & =\tilde{G}-G_{a \neq b}=\frac{1}{G_{c}^{-1}} \\
G_{a \neq b} & =-\frac{G_{a \neq b}^{-1}}{\left(G_{c}^{-1}\right)^{2}}
\end{aligned}
$$

Using these inversion formulas is it easy to solve for (1.57). In $d>2$, $B$ depends on $G_{c}$ only and thus $\sigma_{a \neq b}$ is simply a constant proportional to disorder. Given the gaussian nature of the trial action (1.48) the correlation functions such as the density-density can easily be computed

$$
\overline{\langle\rho(r) \rho(0)\rangle}=\left\langle\rho_{a}(r) \rho_{a}(0)\right\rangle \propto e^{-2 B_{a a}(r)}
$$

Using $(1.59)$ shows that $G_{a a}(q) \sim \mathcal{D} / q^{4}$ leading to a growth

$$
B_{a a}(r) \sim \mathcal{D} r^{4-d}
$$


Although this solution is perfectly well behaved, a stability analysis of the replica symmetric saddle point shows that it is unstable. This can be checked from the eigenvalue $\lambda$ of the replicon mode $[56,58$.

$$
\lambda=1-\frac{8 \hbar \mathcal{D}}{(\pi \alpha)^{2}} e^{-4 \hbar \int \frac{d^{d} p}{(2 \pi)^{d}} G_{c}(p)} \int \frac{d^{d} q}{(2 \pi)^{d}} G_{c}^{2}(q)
$$

A negative eigenvalue $\lambda$ indicates an instability of the replica symmetric solution. We introduce a small regularizing mass in $G_{c}: G_{c}(q)^{-1}=c q^{2}+\mu^{2}$ and take the limit $\mu \rightarrow 0$. It is easy to see from (1.63) that for $d<2$ the replica symmetric solution is always stable. In that case disorder is in fact irrelevant, due to the strong quantum (or thermal for the associated classical system) fluctuations. For $d=2$ the condition becomes $\mu^{2(\bar{K}-1)}<1$ for small $\mu$. Thus there is a transition at $\bar{K}=1$ between a replica symmetric stable high temperature phase where disorder is irrelevant and a low temperature (glassy) phase where the symmetric saddle point is unstable. For the classical system this is the well known Cardy-Ostlund transition [59], with very interesting physical aspects of its own. This transition is the equivalent for time dependent disorder of the localization transition studied in section 3.2. For time dependent disorder one time integral drops in (1.29) and (1.30) would become $(2-2 K) \mathcal{D}$ giving the transition at $K=1$. More details can be found in [58, 60, 57]. For $2<d<4$ the replica symmetric solution is always unstable.

One should thus look for another way of inverting the $0 \times 0$ matrices than the replica symmetric one. Fortunately such a scheme was invented in the context of spin glasses. Instead of having a single value $\sigma$ for the off diagonal term, one introduces a whole set of values. Let us briefly illustrate the procedure here, refering the reader to 61 for details. Let us introduce a set of integers $m_{0}=n, m_{1}, \ldots, m_{k+1}=1$ such that $m_{i} / m_{i+1}$ is an integer. One cuts the matrix in blocks of size $m_{1}$ as illustrated on Figure 4 . Elements outside the blocks have the value $\sigma_{0}$. The procedure is then recursively applied for the inner blocks. At the last step the value on the block of size $m_{k}$ is $\sigma_{k}$ and a diagonal value $\tilde{\sigma}$. Quite generally the matrix is thus now parametrized by a diagonal element and a whole function (in the limit $n \rightarrow 0$ ) $\sigma\left(u\right.$ ), where $u \in[0,1]$ (notice the range of variation of the $m_{i}$ when $n \rightarrow 0$ ). Such matrices can also be inverted in the limit $n \rightarrow 0$ ), albeit with more complicated inversion rules than for the RS solution. When one has a continuous function (see Figure 5) the RSB is said to be continuous. Simpler cases are of course a constant function (a single off-diagonal value) which is simply the RS solution or a function continuous by steps. We have represented in Figure (see also Figure 5) the case of a two-step RSB. We denote $\tilde{G}(q)=G_{a a}(q)$, similarly $\tilde{B}(x)=B_{a a}(x)$, and parametrize $G_{a b}(q)$ by $G(q, v)$ where $0<v<1$, and $B_{a b}(x)$ by $B(x, v)$. Physically, $v$ parametrises pairs of low lying states, in the hierarchy of states, $v=0$ corresponding to 


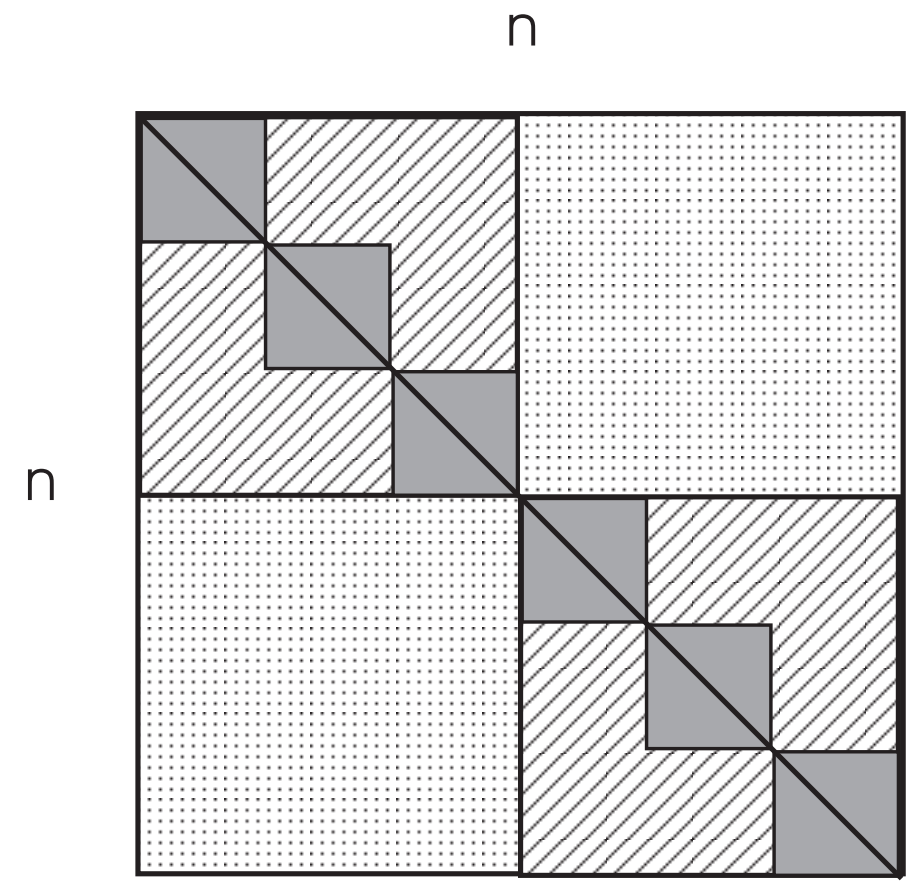

FIGURE 4. Replica symmetry broken matrices (here for a 2-step RSB). Each pattern correspond to a different value $\sigma_{i}$ (see text), and the diagonal one (black line) is $\tilde{\sigma}$. 
states further apart. The saddle point equations become:

$$
\sigma(v)=\frac{2 \mathcal{D}}{(\pi \alpha)^{2}} e^{-2 B(0, v)}
$$

where

$$
B(0, v)=2 \hbar \int \frac{d^{d} q}{(2 \pi)^{d}}(\tilde{G}(q)-G(q, v))
$$

$B(0, v)$ corresponds physically to the mean squared phase fluctuations at the same point in space $(r=0)$ (for the associated classical system this bould be mean squared relative displacements of the same object) but in two replica states, or more physically in two different low lying metastable states. The large distance behaviour of disorder-averaged correlators is determined by the small $v$ behaviour of $B(0, v)$. We look for a solution such that $\sigma(v)$ is constant for $v>v_{c}, v_{c}$ itself being a variational parameter, and has an arbitrary functional form below $v_{c}$. This corresponds to full RSB (see Figure 5). The algebraic rules for inversion of hierarchical matrices 56] give:

$$
B(0, v)=B\left(0, v_{c}\right)+\int_{v}^{v_{c}} d w \int \frac{d^{d} q}{(2 \pi)^{d}} \frac{2 \hbar \sigma^{\prime}(w)}{\left(G_{c}(q)^{-1}+[\sigma](w)\right)^{2}}
$$

where $[\sigma](v)=u \sigma(v)-\int_{0}^{v} d w \sigma(w)$ and

$$
B\left(0, v_{c}\right)=\int \frac{d^{d} q}{(2 \pi)^{d}} \frac{2 \hbar}{G_{c}(q)^{-1}+[\sigma]\left(v_{c}\right)}
$$

This is a simple number. Taking the derivative of (1.64) with respect to $v$, using $[\sigma]^{\prime}(v)=v \sigma^{\prime}(v),(1.66)$, and (1.64) again one finds

$$
1=\sigma(v) \int \frac{d^{d} q}{(2 \pi)^{d}} \frac{4 \hbar}{\left(c q^{2}+[\sigma](v)\right)^{2}} \simeq \sigma(v)\left(\frac{4 \hbar c_{d}}{c^{d / 2}}\right)[\sigma(v)]^{(d-4) / 2}
$$

Since the integral is ultraviolet convergent, we have taken the short-distance momentum cutoff to infinity. $c_{d}$ is a simple number

$$
c_{d}=\int \frac{d^{d} q}{(2 \pi)^{d}}\left(\frac{1}{q^{2}+1}\right)^{2}=\frac{(2-d) \pi^{1-d / 2}}{2^{d+1} \sin (d \pi / 2) \Gamma(d / 2)}
$$

with $c_{d=3}=1 /(8 \pi), c_{d=2}=1 /(4 \pi)$. Derivating one more time one gets for the effective self energy:

$$
[\sigma](v)=\left(u / u_{0}\right)^{2 / \theta}
$$

where $\theta=(d-2)$ and $v_{0}=8 \hbar c_{d} c^{-d / 2} /(4-d)$. The shape of $[\sigma](u)$ is shown on Figure 5. The solution (1.70), is a priori valid up to a breakpoint $u_{c}$, 


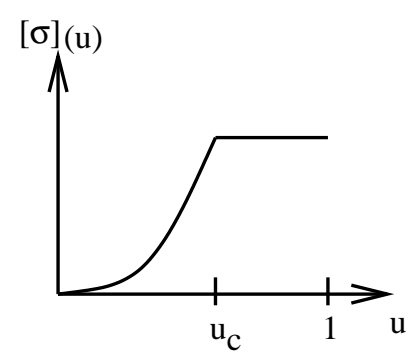

(a)

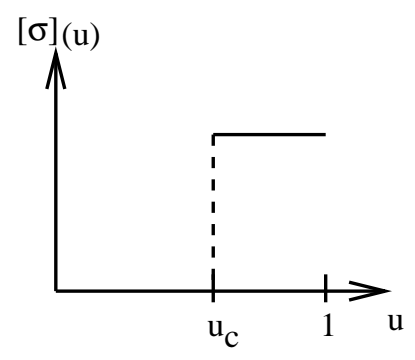

(b)

FIGURE 5. Shape of the self energy $\sigma$ as a function of $u$. (a) is the full RSB solution occuring for $2<d<4$. (b) a one step $\operatorname{RSB}(d=2)$.

above which $[\sigma]$ is constant, since $\sigma^{\prime}(u)=0$ is also a solution of the variational equations. $u_{c}$ can also be extracted from the saddle point equations and we refer the reader to [58] for details. The precise value of $u_{c}$ is unimportant for our purpose but the existence of the two distinct regimes in $[\sigma](u)$ has a simple physical interpretation that we examine in section 6.2 Using $(1.70)$ one can now compute the correlation functions. Larger distances correspond to less massive modes and is dominated by the small $u$ behavior of (1.70). One obtains

$$
\begin{aligned}
\overline{\left\langle(\phi(r)-\phi(0))^{2}\right\rangle} & =2 \hbar \int \frac{d^{d} q}{(2 \pi)^{d}}(1-\cos (q r)) \tilde{G}(q) \\
\tilde{G}(q) & =\frac{1}{c q^{2}}\left(1+\int_{0}^{1} \frac{d v}{v^{2}} \frac{[\sigma](v)}{c q^{2}+[\sigma](v)}\right) \sim \frac{Z_{d}}{q^{d}}
\end{aligned}
$$

with $Z_{d}=(4-d) /\left(4 \hbar S_{d}\right)$ and $1 / S_{d}=2^{d-1} \pi^{d / 2} \Gamma[d / 2]$. Thus for $2<d<4$ this leads to a logarithmic growth,

$$
\overline{\left\langle(\phi(x)-\phi(0))^{2}\right\rangle}=\frac{1}{2} A_{d} \log |x|
$$

with $A_{d}=4-d$, instead of the power law growth (1.62) of the replica symmetric solution. Note that the amplitude is independent of disorder.

\subsection{If it ain't broken ...}

It is thus necessary to break the replica symmetry to get the correct asymptotic physics $\left(1 / q^{d}\right.$ propagator) instead of the $1 / q^{4}$ given by the replica symmetric solution. From (1.72) one easily sees that at large enough $q$ (i.e. for short distances) one recovers the replica symmetric solution. Thus $u_{c}$ and $\left[\sigma\left(u_{c}\right)\right]$ (see figure 5 ) define a lengthscale $L \sim\left(c /\left[\sigma\left(u_{c}\right)\right]\right)^{1 / 2}$ above which the RS solution does not describe the physics. This lengthscale corresponds to the pinning length similar to (1.24) obtained by balancing the 
elastic energy with the disorder one. Here

$$
\begin{aligned}
E_{\mathrm{el}} & \sim L^{d-2} \\
E_{\mathrm{dis}} & \sim \mathcal{D}^{1 / 2} L^{d / 2}
\end{aligned}
$$

leading to the famous Larkin [62, 63] length $L_{\text {loc }} \sim(1 / \mathcal{D})^{1 /(4-d)}$ which corresponds to the distance for which relative displacements are of the order of the lattice spacing [64] (or for which the phase here is of the order $2 \pi$ ) 65. Below this lengthscale the system has a single equilibrium state. It can be described by simply expanding in the displacements in (1.13)

$$
H_{\mathrm{dis}}=\int d^{d} x f(x) \phi(x)
$$

where $f$ is a random force, derivative of the random potential $V$. It is easy to see that this model gives the $1 / q^{4}$ propagator. This breaks down when the displacements cannot be expanded, i.e. for distances larger than $L_{\mathrm{loc}}$. As anticipated by Larkin and Ovchinikov [63, pinning occurs that tends to keep a domain of size $L_{\text {loc }}$ in place despite the thermal fluctuations.

This help us to understand the physics of the RSB solution. To illustrate it let us focus for simplicity to the case in $d=2$. As can be seen from (1.70) by letting $d \rightarrow 2$ the RSB solution in that case is a one-step breaking as shown in Figure . There are some additional complications but they are unimportant for our discussion here. A normal self consistent approximation would approximate the energy by a simple Gaussian centered in $\phi=0$. The only way to incorporate the pinning is thus to put a mass term in the propagator

$$
q^{2} \rightarrow q^{2}+L_{\mathrm{loc}}^{-2}
$$

As can be immediately inferred from the discussion of section 3.1, this is a much too crude approximation. The RSB solution is smarter and approximates effectively the distribution of displacements by a hierarchical superposition of Gaussians centered at different randomly located points in space. A pictorial view of these two cases is shown on Figure 6. The double distribution over environment and thermal (or quantum) fluctuations is approximated as follows [57. In each environment there are effective "pinning centers" corresponding to the low lying metastable states (preferred configurations). Since all $q$ modes are in effect decoupled within this approximation, for each $q$ mode a preferred configuration (a state) is $\phi_{\alpha}(q)$. They are distributed according to:

$$
P\left(\left\{\phi_{\alpha}(q)\right\}\right) \sim \prod_{\alpha} e^{-\frac{c}{2 T_{g}} q^{2}\left|\phi_{\alpha}(q)\right|^{2}}
$$

Each is endowed with a free energy $f_{\alpha}$ distributed according to an exponential distribution $P(f) \sim \exp \left(u_{c} f / T\right)$ (here $\left.u_{c}=T / T_{g}\right)$. Once these seed 
(A)

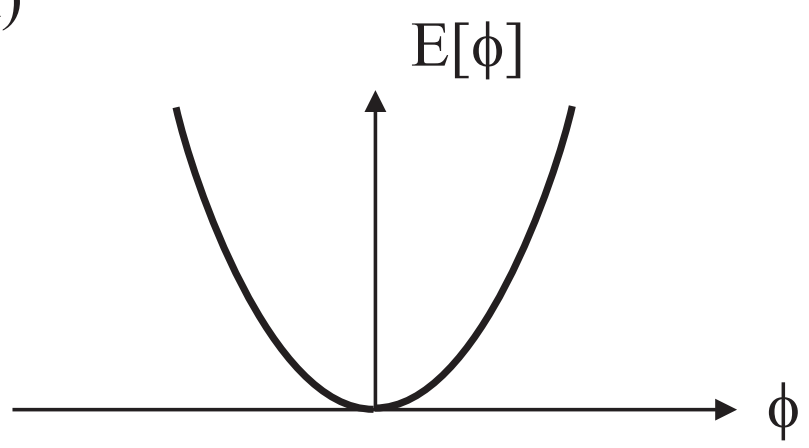

(B)

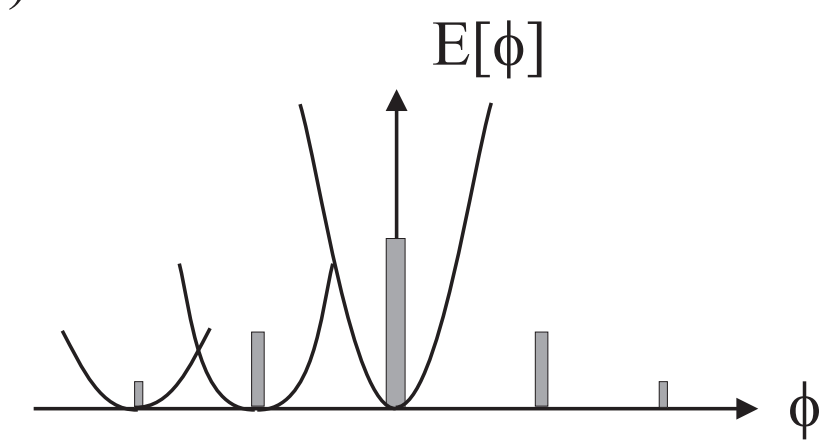

FIGURE 6. (A) The RS solution describes fluctuations around a single state, with a single spring constant. (B) the RSB solution takes correctly into account the fact that different regions of space correspond to different equilibrium values of $\phi$, with a priori different spring constants. The weight of a given configuration has been represented by the height of the shaded region. 
states are constructed, the full thermal distribution of the $q$ mode $\phi_{q}$ is obtained by letting it fluctuate thermally around one of the states:

$$
P\left(\phi_{q}\right) \sim \sum_{\alpha} W_{\alpha} e^{-\frac{c}{2 T}\left(q^{2}+L_{\mathrm{loc}}^{-2}\right)\left|\phi_{q}-\phi_{\alpha}(q)\right|^{2}}
$$

where each state is weighted with probability $W_{\alpha}=e^{-f_{\alpha} / T} / \sum_{\beta} e^{-f_{\beta} / T}$. One thus recovers qualitatively the picture of Larkin Ovchinikov as the solution of the problem with the replica variational method. The Larkin length naturally appears as setting the (internal) size of the elastically correlated domains. The full RSB case corresponds to more levels in this hierarchy of Larkin domains (in some sense there are clusters of domains of size larger than $L_{\mathrm{loc}}$ ) and the way this hierarchy scales with distance reproduces the exponents for displacements and energy fluctuations. We refer the reader to [57 for more details on the use of the variational method in this context and for the physical properties of such systems.

\subsection{Quantum problems}

We now apply the same method to the quantum problem. Because of the non locality in time of the interaction in (1.46) the solution will have quite different properties. We again use the trial action (1.48), with the parametrization

$$
v G_{a b}^{-1}\left(q, \omega_{n}\right)=\frac{\left((v q)^{2}+\omega_{n}^{2}\right)}{\pi K} \delta_{a b}-\sigma_{a b}\left(\omega_{n}\right)
$$

Although, as in section 6.1 $\sigma_{a b}$ is still independent of $q$ because of the locality in space it is now dependent on $\omega_{n}$. This would render the solution extremely complicated if it were not for a remarquable property of quantum systems. Off diagonal replica terms such as $\sigma_{a \neq b}$ only exist for the mode $\omega_{n}=0$ The general argument [66, 67] is that in each realization of the random potential $V$, the disorder does not depend on $\tau$. Therefore before averaging over disorder:

$$
G_{a b, V}=\left\langle\phi_{a}(x, \tau) \phi_{b}(0,0)\right\rangle=\left\langle\phi_{a}(x, \tau)\right\rangle\left\langle\phi_{b}(0,0)\right\rangle=\left\langle\phi_{a}(x, 0)\right\rangle\left\langle\phi_{b}(0,0)\right\rangle
$$

It is important to note that such a property crucially depends on the assumption that the hamiltonian is $\tau$-independent and on the fact that equilibrium has being attained. This is the case considered here.

The static mode $\omega_{n}=0$ thus plays a special role. This is quite natural in a time independent disorder. Quite naively one sees already that the properties of the variational solution will thus be very similar to the ones of point like (i.e. totally uncorrelated) disorder in $d$ spatial dimensions (here $d=1$ ). This suggest strongly that the variational method will pull out a static solution, reminiscent of the one introduced in section 3.1 
and treat the fluctuations around this static solution. We will come back to this point later. The solution can be obtained quite in all dimensions and we refer the reader to [67] for details. We specialize here to the case $d=1$. In this case two type of solution exist, a simple RS solution with $\sigma_{a b}=0$. This solution is stable for $K>3 / 2$. It corresponds of course to the delocalized regime where the cosine term in (1.46) is irrelevant. The variational method correctly reproduces the (gaussian) delocalized regime, and the correct transition point, but of course misses the renormalization of the Luttinger parameters given by the RG. For $K<3 / 2$ although an RS solution still exists it is unstable and physically obviously incorrect 67. One should look for an RSB solution. In that case the correct solution is a one-step RSB solution (it would be full RSB for $d>2$ ) of the type shown in Figure 5 .

$$
\begin{aligned}
G_{c}^{-1}\left(q, \omega_{n}\right)= & \frac{\hbar}{\pi K}\left(v q^{2}+\frac{\omega_{n}^{2}}{v}\right)+\frac{2 \mathcal{D}}{\hbar(\pi \alpha)^{2}} \int_{0}^{\beta \hbar} d \tau\left(1-\cos \left(\omega_{n} \tau\right)\right) \\
& {\left[\exp (-2 \hbar \tilde{B}(x=0, \tau))-\int_{0}^{1} d u \exp (-2 \hbar B(u))\right](1.82) }
\end{aligned}
$$

with

$$
\sigma\left(q, \omega_{n}, u\right)=\frac{2 \mathcal{D} v}{(\pi \alpha)^{2}} \beta \exp (-\hbar 2 B(u)) \delta_{\omega_{n}, 0}
$$

These equations are still formidable to solve. A simple parametrization is

$$
\begin{array}{r}
v G_{c}^{-1}\left(q, \omega_{n}\right)=\frac{1}{\pi \bar{K}}\left((v q)^{2}+\omega_{n}^{2}\right)+\Sigma_{1}\left(1-\delta_{n, 0}\right)+I\left(\omega_{n}\right) \\
I\left(\omega_{n}\right)=\frac{2 \mathcal{D} v}{(\pi \alpha)^{2} \hbar} \int_{0}^{\beta \hbar}\left[e^{-2 \hbar \tilde{B}(\tau)}-e^{-2 \hbar B\left(u>u_{c}\right)}\right]\left(1-\cos \left(\omega_{n} \tau\right)\right) d \tau \\
\Sigma_{1}=u_{c}\left(\sigma\left(u>u_{c}\right)-\sigma\left(u<u_{c}\right)\right)=[\sigma]\left(u>u_{c}\right) \\
\sigma(u)=\frac{2 \mathcal{D} v}{(\pi \alpha)^{2}} e^{-\hbar 2 B(u)} \beta \delta_{n, 0}
\end{array}
$$

The parameters $\Sigma_{1}$, the breakpoint $u_{c}$ and the function $I\left(\omega_{n}\right)$ have to be determined self-consistently. Let us examine first the general properties of the solution.

Since $I\left(\omega_{n}=0\right)=0$ it is easy to check from (1.16) that the compressibility is unchanged by the disorder, since the "mass" term $\Sigma_{1}$ goes also away at $\omega_{n}=0$. The variational method thus correctly reproduces that the compressibility of an Anderson insulator is still finite, and practically unchanged (for free electrons where we can compute it) from the value without disorder. Correlation functions are also easy to obtain. We just give here the important physical point without the explicit derivation 67. Because of the presence of $\Sigma_{1}$ in the propagator (1.84) they will be massive. This leads to

$$
B(x \rightarrow \infty, \tau=0) \rightarrow x / L_{\mathrm{loc}}
$$


A full calculation of the correlations shows that $\tilde{B}(\tau)$ grows until $\tau \sim L_{\text {loc }}$ when it saturates

$$
B(x=0, \tau \rightarrow \infty) \rightarrow \text { Cste }=\left(\rho_{0}^{-1} l_{\perp}\right)^{2}
$$

Since the variational action is gaussian one has for the correlation of the $2 k_{F}$ part of the density

$$
\chi_{\rho}(x, \tau)=e^{-2 B(x, \tau)}
$$

Two different physical effects are described by the above correlation functions. In the absence of disorder $\chi_{\rho}(\tau \rightarrow \infty)$ would go to zero as a power law, a sign of the wandering of the particles. (1.89) traduces the fact that the time independent potential localizes the particles at a given point in space, instead of letting them fluctuate (unboundedly) due to quantum fluctuations in the absence of disorder. This leads to density correlation in time going to a constant up to a Debye Waller like factor. The behavior (1.89) thus shows that a static solution $\phi_{0}(x)$ exists. The variational approach thus provides a good justification for the static solution $\phi_{0}$ on which the method of section 3 is built. The correlation length of this static solution is given by (1.88). Since this length controls through (1.90) the exponential decay of the spatial correlations of density it is thus related to the standard localization length. Note that here both $L_{\mathrm{loc}}$ and the full static solution are determined by the variational method. Simple dimensional analysis on (1.84) shows that

$$
L_{\mathrm{loc}} \propto 1 / \sqrt{\Sigma_{1}} .
$$

The solution of the variational equations [67] leads back to the expression (1.28) for $L_{\text {loc }}$. Quite interestingly (1.89) defines a length if one writes the constant in units of the fermion spacing. This length is the "width" of the fluctuations of the particles as shown in Figure 7. Note the difference between the two lengths $l_{\perp}$ and $L_{\mathrm{loc}}$. They are related through 67]

$$
l_{\perp}^{2}=\frac{\alpha^{2}}{\pi^{2}}(\hbar \bar{K}) \ln \left(L_{\mathrm{loc}} / a\right)
$$

At the transition one expects that $L_{\mathrm{loc}}$ diverges as $L_{\mathrm{loc}} \sim \exp \left(b /\left(K_{c}-K\right)^{\alpha}\right)$ with $\alpha=1 / 2$ from the RG (see equation (1.35)). Thus relation (1.92) predicts that:

$$
l_{\perp} \sim \frac{1}{\left(K_{c}-K\right)^{\alpha / 2}}
$$

diverges as a power law, which could be measured in numerical simulations.

Let us now look at the conductivity, for which we need $I\left(\omega_{n}\right)$. If we call $I^{\prime}(\omega)$ and $I^{\prime \prime}(\omega)$ the real and imaginary parts of the analytic continuation of $I\left(\omega_{n}\right)$, then the conductivity is given by

$$
\sigma(\omega)=\frac{\omega I^{\prime \prime}+\omega\left(-\omega^{2}+I^{\prime}+\Sigma_{1}\right)}{\left(-\omega^{2}+\Sigma_{1}+I^{\prime}\right)^{2}+\left(I^{\prime \prime}\right)^{2}}
$$




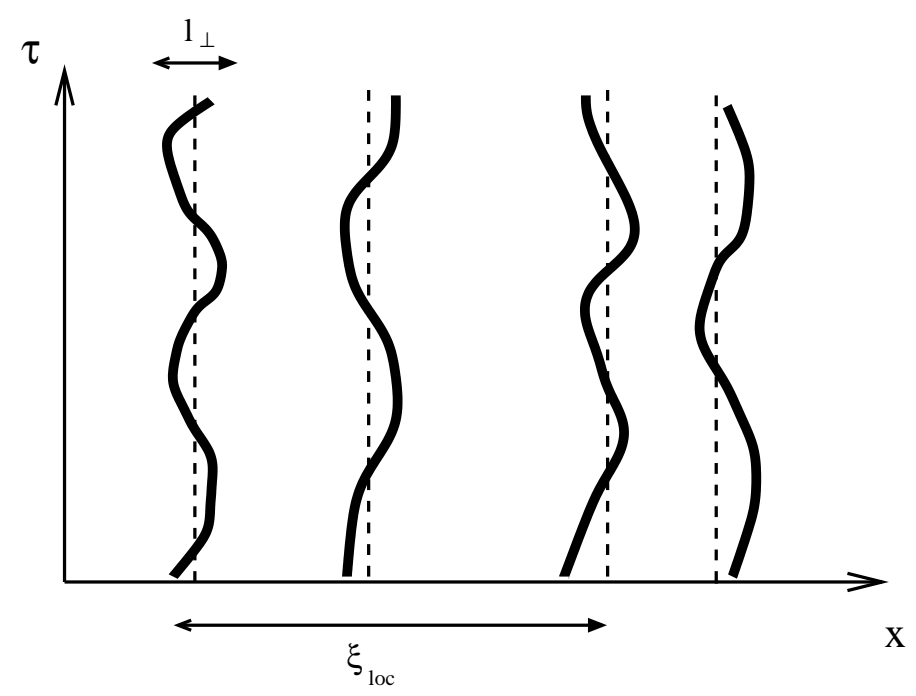

FIGURE 7. Localization.

It is easy to see that $\Re \sigma(\omega)$ goes to zero at zero frequency and thus the phase is indeed localized. But because of the analytic continuation, the existence or not of a gap in the optical conductivity is not linked to the existence of a "mass" $\Sigma_{1}$ but to whether $I^{\prime \prime}$ is nonzero at small frequencies or not. The equation for $I\left(\omega_{n}\right)$ takes a particularly simple form in the limit $K, \hbar \rightarrow 0$ while keeping $\bar{K}$ fixed. In this limit, we can write

$$
I\left(\omega_{n}\right)=\Sigma_{1} f\left(\frac{\omega_{n}}{\sqrt{\pi \bar{K} \Sigma_{1}}}\right)
$$

where the scaling function $f$ satisfies:

$$
f(x)=2\left[1-\frac{1}{\sqrt{1+x^{2}+f(x)}}\right] .
$$

It can be shown that for $\omega \rightarrow 0$

$$
\Re \sigma(\omega) \sim \omega^{2}
$$

In a similar way (1.94) gives

$$
\Im \sigma(\omega) \sim \omega / \Sigma_{1}
$$

Such behavior is in agreement with exact results in one dimension [2, 3] up to logarithmic prefactors. At high frequency, one can show that $\sigma(\omega) \sim$ $\omega^{2 K-4}$ in agreement with the RG result. The resulting conductivity is plotted on Figure 8 . 


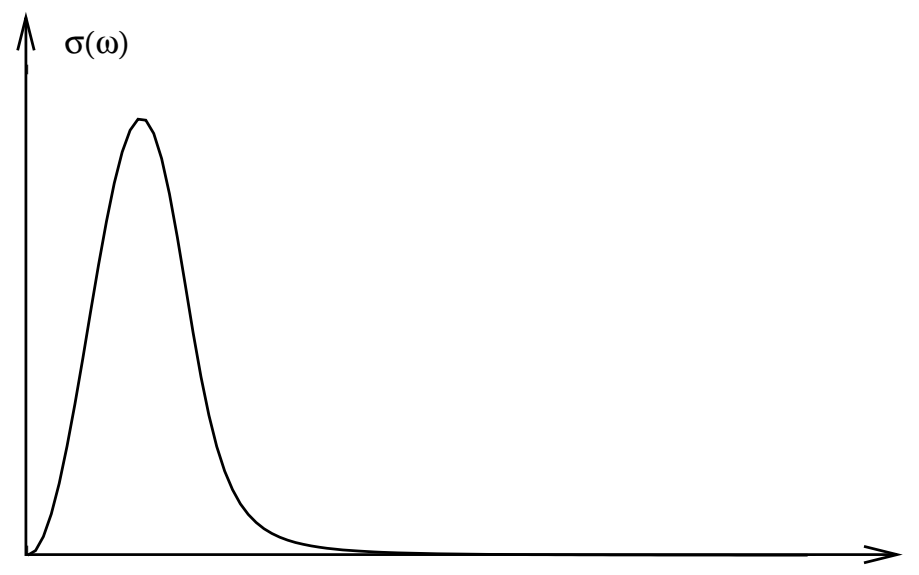

FIGURE 8. The conductivity of the one dimensional Anderson insulator in the limit $K \rightarrow 0, \hbar \rightarrow 0, \bar{K}=\frac{K}{\hbar}$ fixed.

\subsection{The fine prints}

The variational method is thus an extremely efficient method for this type of disordered problems. It allows to get most of the physical properties in the localized phase, and give the qualitative features of the transition and the delocalized phase. Its physics is very similar to the one described for point like disorder in section 6.1. The variational method determines the "static" solution for the mode $\omega_{n}=0$ (but by taking into account the effects of all modes) without being restricted to a single Gaussian. It can then correctly compute the fluctuations (both in $q$ and $\omega_{n}$ ) around this solution, contrarily to the approximate method of section 3.1 which was impeded by the lack of knowledge of the static solution.

In $d=1$ an additional remarkable property can be seen. Although most of the properties are independent of the value of $u_{c}$, the conductivity is strongly dependent on it. As for the static solution one has continuous replica symmetry breaking for $d>2$ which goes continuously to the one step solution in $d=2$. In these dimensions taking the value of $u_{c}$ out of the variational equations gives the correct conductivity (no gap in $\sigma(\omega)$ ). In $d=1$, the $u_{c}$ obtained by minimizing the free energy would give an incorrect (gapped) conductivity. Another way to determine $u_{c}$ is to use the marginality condition [67] that corresponds to the instability of the replicon mode, similar to the condition (1.63). This condition, more dynamical in nature, coincides with the free energy value of $u_{c}$ for $d \geq 2$ but is different in $d=1$. One can check that the marginality condition always gives the correct conductivity. Some arguments for why it is so were given in 67, but this point is not yet fully understood. This phenomenon has since been found to occur in other systems 68. 

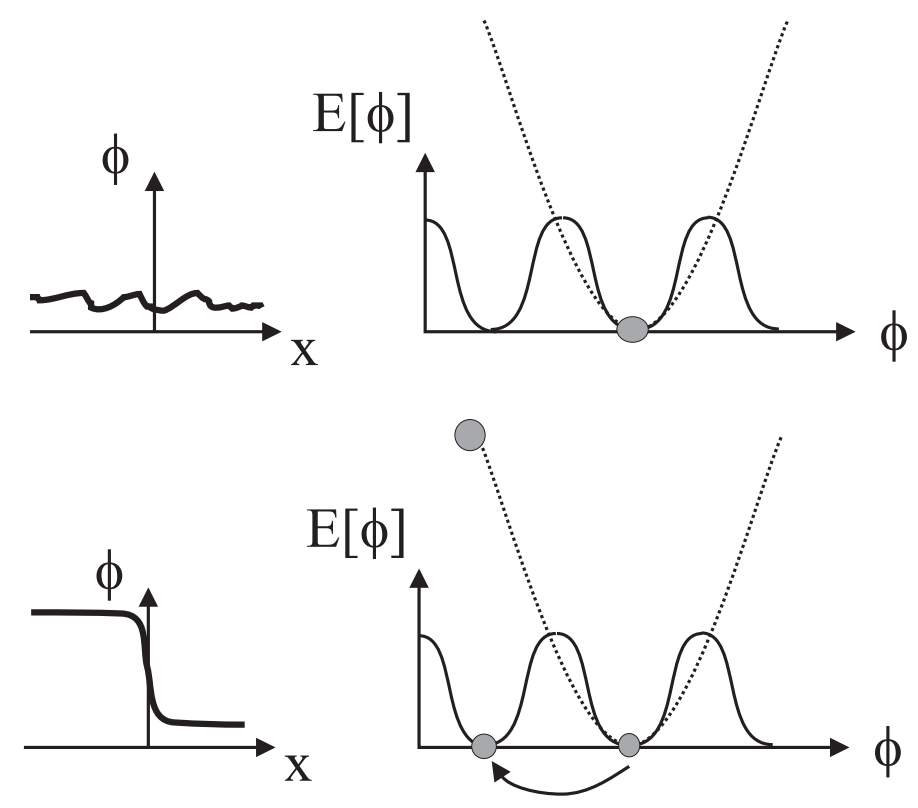

FIGURE 9. The gaussian approximation treats correctly the excitations where the phase stays in one of the minima of the cosine. They contribute to the a. c. conductivity at $T=0$. Soliton excitations bring the phase from one minimum to another. With the $\cos (\phi)$ term these excitations only cost energy at the kink. When the potential is replaced by the quadratic approximation, the energy cost of the "wrong" minimim is very high, hence a poor result for the physical results. These excitations dominate the d.c. conductivity for $T>0$.

The success of the variational method is obviously linked to the fact that we are looking at small "quadratic" fluctuations around a certain (in our case highly disordered) solution. In this case the replacement

$$
-\cos (\phi) \rightarrow \frac{1}{2} \phi^{2}
$$

is very reasonable. Such an approximation is very good to compute correlation functions of the variable $\phi$. This is the case for the density-density correlation and the optical conductivity. What is missing in the approximation (1.99) are the solitons that go from one minimum of the potential to another minimum. The energy cost of such excitations is grossly overestimated by the variational method as is shown on Figure 9 .

This has important consequences for the calculation of correlation functions that involve the soliton creation operator $e^{i \theta}$. These correlation functions are found incorrectly to decay exponentially with distance at equal time and to be zero at unequal time. As a result, the variational method does not allow the calculation of Fermion Green's function (they involve the operators $e^{i(\theta \pm \phi)}$ ) nor superconducting correlations (which involve the 


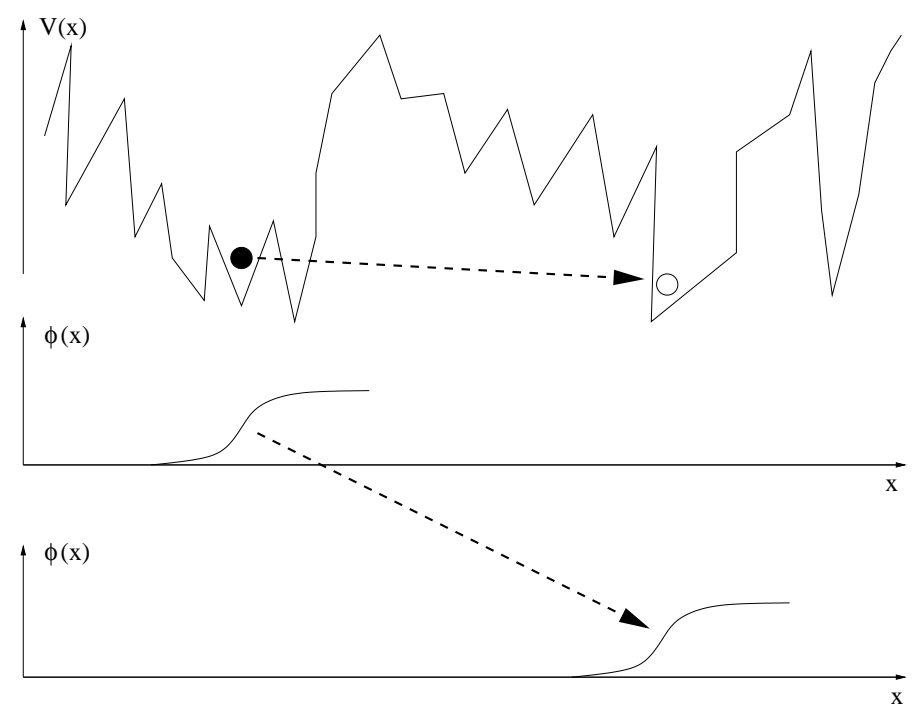

FIGURE 10. Hopping of charges that leads to a finite d.c. conductivity. This corresponds to soliton-type excitations in the phase variable $\phi$.

operator $\left.e^{2 i \theta}\right)$. In the case of a disordered XXX spin chain, the situation is even worse, since $S_{x} \propto \cos (\theta)$, whereas $S_{z} \sim-\frac{\partial_{x} \phi}{\pi}+(-)^{x / a} \cos 2 \phi$. Therefore, even in the presence of a disorder that preserves $\mathrm{SU}(2)$ symmetry (such as a random bond disorder), the variational method would lead to a spurious breaking of rotational symmetry. It would also give poor results for systems that include both the $\theta$ and the $\phi$ field in the Hamiltonian. Examples of such theories include disordered Hubbard ladders [43], disordered spin ladders [46] or XXX spin chain in a random fields [22].

The mishandling of soliton excitations also limits our knowledge of the transport properties at finite temperatures. Indeed the optical conductivity does not correspond to transport of charge but charge oscillations around the equilibrium positions, it is thus well described by our harmonic approximation as shown in Figure 9. On the contrary transport at finite $T$ involves real charge displacements. Since $\rho(x) \sim \nabla \phi$, displacing a charge amounts to make a solitonic excitation in the field $\phi$ as shown in Figure 10. Indeed Mott's arguments, to compute the conductivity of noninteracting electrons in presence of phonons $\sigma(T) \sim \exp \left[-\left(\frac{T_{0}}{T}\right)^{\frac{1}{d+1}}\right]$ is strongly reminiscent of an instanton calculation as shown in Figure 10. A similar argument applies to Efros and Schklovskii calculations 69. An extension of the variational approach to finite temperatures would indeed still lead to $\sigma(T, \omega=0)=0$ proof that it is missing the excitations that are important at finite temperature. Unfortunately no way to treat such solitons has been found at present despite some attempts 70].

Despite these limitations, the variational method is up to now the only 
analytical method giving information for such localized systems in the localized phase. As with all variational approaches, some physical insight in the properties of the system under consideration is needed to determine whether the method as any chance of success. Clearly, one must apply this method only to systems that can be reasonably well understood qualitatively from their classical action. Fortunately many systems fall in this category, and we examine some of those in the following.

\subsection{Higher dimension: electronic crystals and classical systems}

First the GVM can be used to study classical systems using the standard mapping $\tau \rightarrow z$. The action (1.46) and its extension to higher spatial dimension describes elastic objects (lines in this case as shown in Figure 11) pinned by columnar (i.e. time or $z$ independent) defects. This situation is realized for example in vortex in type II superconductors irradiated by heavy ions (creating the linear track of disorder). This system in $2+1$ dimension is equivalent to a $d=2$ quantum bose system in presence of pins. In a similar way than in $d=1$ (see section 6.3) such system has a pinned phase (the Bose glass) 21, 71. The variational method can be used to describe the Bose glass phase 67]. However contrarily to $d=1$ it cannot be used to go to the superfluid regime since to describe a two dimensional "melting" of the Bose glass phase dislocations are important (no dislocations exist in $d=1$ ) and for reasons explained above the GVM overestimates the energy cost of topological excitations. Another way to say it, is that in $d>1$ we loose the elastic description (1.39) of the Fermion or Boson operators. The GVM can thus only be used in phases where the particles are localized so that some elastic description can again be used. We can thus use the variational method in higher dimensions to study electronic crystals. This included Charge density wave, but also the two dimensional Wigner crystal of electrons. In such a phase the electrons are confined by their repulsion (and a in some systems a magnetic field). An elastic description can be used. Some level of quantumness is hidden in the elastic parameters ("size" of the particles, quantization of the phonon modes of the crystal). For such systems the calculation of the optical conductivity is particularly useful since it is one of the few probes of such systems. Since the physics of such systems would deserve a review of its own we will not dwell further on it here but refer the reader to 72,73 for details.

\section{Commensurate systems}

When the filling of the fermion system is commensurate the physics discussed above is modified in various ways since the backward scattering on 


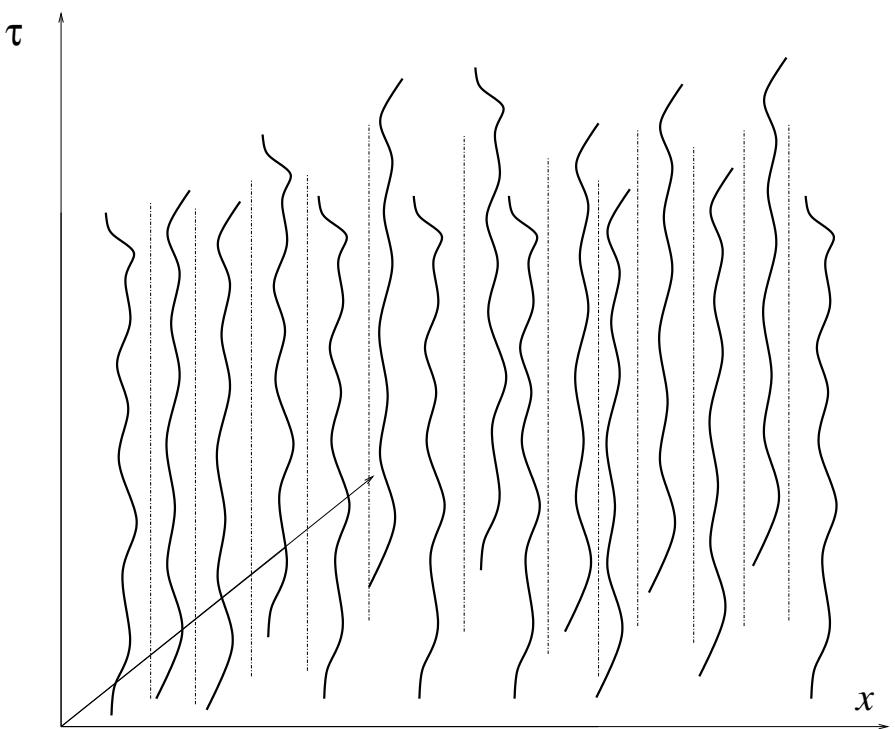

FIGURE 11. The action 1.46 in $d=2$ describes elastic lines in the presence of columnar pins.

disorder becomes real. If the forward scattering still exists, not much is changed. Two special cases will thus occur: (i) the forward scattering is absent. This occurs because of a symmetry of the system. This is the case for example for fermions at half filling with a random hopping or for spin chains with random exchange. (ii) The commensurate potential (either due to the lattice or due to electron-electron interaction) would open a gap. There will be a competition between Mott physics wanting to get a commensurate (gapped) insulator and the disorder that would like to destroy such a gap (push the system locally away from commensurability).

\subsection{The peculiar random exchange}

For electrons at half filling with a random exchange the forward scattering does not exist and the disorder term is simply

$$
\begin{aligned}
H & =\int d x V(x) i\left[\psi_{+}^{\dagger} \psi_{-}-\text {H.c. }\right] \\
& =-\int d x \frac{V(x)}{(2 \pi \alpha)} \sin (2 \phi(x))
\end{aligned}
$$

Although this seems very similar to (1.13) one easily sees the difference on Figure 12. Contrarily to normal disorder where $\phi$ follows the random phase of the random potential, here $\phi= \pm \pi / 4$ depending on the sign of the potential. Thus $\phi$ is nearly gapped but for its kinks. The low energy 


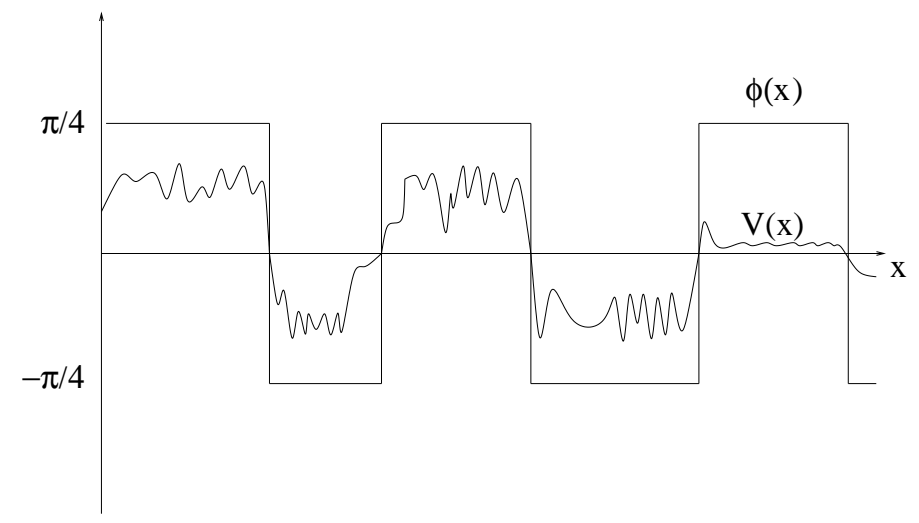

FIGURE 12. The profile of $\phi(x)$ for a given realization of disorder $V(x)$. As one can see, $\phi(x)$ is only sensitive to the sign of $V(x)$ and not to its amplitude. As a result, $\phi$ has kinks each time $V(x)$ goes to zero.

properties will thus be dominated by the kinks in $\phi$. Such kink structure between the doubly degenerate minima makes it unlikely that the GVM can be used for this problem.

In order to get an idea of the physics let use examine the case of free fermions $K=1$. For $E=0$ one can easily construct the eigenstates by solving

$$
\begin{gathered}
\partial \psi_{+} \quad-\quad V(x) \psi_{-}(x)=0 \\
-\partial \psi_{-}+i V(x) \psi_{+}(x)=0
\end{gathered}
$$

to obtain the (unnormalized)

$$
\psi_{+}(x) \sim \psi_{-}(x) \sim e^{-\int_{0}^{x} d y V(y)}
$$

which obviously decays as

$$
\psi_{ \pm}(x) \sim e^{-\sqrt{x}}
$$

If one wants to view such a state as an exponentially localized state this means that the localization length diverges when $E \rightarrow 0$. The divergence of the localization length is of course the signature that the physical properties will be quite different than the ones for the "normal" disorder studied in the previous sections. For the non-interacting case they have been studied using a variety of techniques such as replicas, Berezinskii methods, supersymmetry etc. [74, 75, 76, 77]. The density of states diverge at the Fermi level as 78

$$
\rho(\epsilon) \sim \frac{1}{\epsilon \log (1 / \epsilon)^{3}}
$$

Quite astonishingly if one considers that all states are still localized the d.c. conductivity is now finite [74, 79]. This is surprising since it seems to violate 
the Mott phenomenological derivation. The conductivity is proportional to the absorbed power. To make a transition between two localized states one need one occupied state, one empty one separated by an energy $\hbar \omega$. The number of such states is $\rho(\epsilon=0) \hbar \omega$. The absorbed power (and the conductivity) is thus (up to log correction)

$$
\sigma(\omega) \propto \rho(\epsilon=0)(\hbar \omega)^{2}
$$

correctly giving back for a constant density of states at the Fermi level the $\omega^{2}$ dependence of section 6.3. One would thus naively have expected a $\sigma(\omega) \sim \omega$ in the case of a singular density of state (1.106). A qualitative understanding of the behavior of the d.c. conductivity is thus still lacking.

When interactions are included, the problem becomes more difficult to tackle. Going back to the spin chain version of (1.100) a real space renormalization procedure has been introduced. This procedure works beautifully and allows the calculation of most correlation functions. We refer the reader to 80, 81] for details. As for the normal disorder (see section 3.2) interaction were found to be irrelevant at the disordered fixed point. The divergence of the localization length also changes drastically the correlation functions. In particular the spin spin correlation functions now decay as power law instead of being exponential. It would be extremely interesting to have an equivalent derivation of this real space RG directly in the boson representation.

\subsection{Mott versus Anderson}

A particularly interesting situation occurs when the non-disordered system possesses a gap. In that case the competition between this gap and the disorder is non trivial. This arises in a large number of systems such as disordered Mott insulators 82, 83, 84, 85, systems with external (Peierls or spin-Peierls systems 86, or internal commensurate potential (ladders or spin ladders [37, 88, 89, 90], disordered spin 1 chains [91, 42]). It is physically simple to see that in order to destroy the gap one needs a disorder comparable to the gap (although in some specific cases Imry-Ma effects can destroy the gap for infinitesimal disorder [82]). This makes the complete description of the gap closure and of the physics of the resulting phases is extremely difficult with the usual analytic techniques such as the perturbative renormalization group described in section 3.2, due to the absence of a weak coupling fixed point. Indeed both the commensurate Mott insulator (which can be described by a sine-Gordon Hamiltonian [92]) and the disordered Anderson insulator correspond to strong coupling fixed points.

To be concrete, but keep the technical details to a minimum let us consider again spinless fermions. The commensurability can be described by adding to the Hamiltonian (1.9) a periodic potential at the Fermi level

$$
H_{\mathrm{com}}=g \int d x\left(\psi_{+}^{\dagger} \psi_{-}+\psi_{-}^{\dagger} \psi_{+}\right)
$$


which becomes in the boson representation

$$
H_{\mathrm{com}}=\int d x \frac{g}{\pi \alpha \hbar} \cos 2 \phi
$$

The reason to consider here a periodic potential is specific to pathologies associated to the Mott gap for spinless fermions [82]. Similar results are expected for a Mott insulator (which is due to the $4 k_{F}$ potential of the lattice).

If this system is studied by RG then one is faced with the competition of two strong coupling fixed points since

$$
\begin{aligned}
\frac{d g}{d l} & =(2-K) g \\
\frac{d \mathcal{D}}{d l} & =(3-2 K) \mathcal{D}
\end{aligned}
$$

Using the usual qualitative argument consisting in taking the most divergent operator, it was concluded [83 that if disorder reaches strong coupling $\left(\mathcal{D}\left(l^{*}\right)=1>g\left(l^{*}\right)\right)$ first, we will be in the Anderson phase, with a localization length $l_{0}=a e^{l^{*}} \sim\left(\frac{1}{\mathcal{D}}\right)^{\frac{1}{3-2 K}}$, whereas if the commensurate potential reaches strong coupling first, we will be in the Mott phase with a correlation length (or soliton size) $d \sim\left(\frac{1}{g}\right)^{\frac{1}{2-K}}$. The phase transition between the two phases occurs for $l_{0} \sim d$. This picture relies on the important assumption that there is no other stable fixed point than the Mott insulator and the Anderson insulator. Even if it was so, it would not be possible to determine the phase boundaries, nor determine what type of critical point separate the Mott and the Anderson insulator. In order to make progress on these issues and obtain a more complete picture of dirty one-dimensional Mott insulators, one needs to solve the problem non-perturbatively 93. The methods of section (6.3) are well adapted since the problem can be cast in the sine-Gordon form.

\subsection{Variational approach}

For the Mott versus Anderson problem, the variational action reads:

$$
\begin{aligned}
\frac{S_{\text {rep. }}}{\hbar} & =\sum_{a}\left[\int \frac{d x d \tau}{2 \pi K}\left(v\left(\partial_{x} \phi_{a}\right)^{2}+\frac{\left(\partial_{\tau} \phi_{a}\right)^{2}}{v}\right)-\frac{g}{\pi \alpha \hbar} \int d x d \tau \cos 2 \phi_{a}\right] \\
& -\frac{\mathcal{D}}{(2 \pi \alpha \hbar)^{2}} \sum_{a, b} \int d x \int_{0}^{\beta} d \tau d \tau^{\prime} \cos \left(2\left(\phi_{a}(x, \tau)-\phi_{b}\left(x, \tau^{\prime}\right)\right)\right)(1.112)
\end{aligned}
$$

The term $\cos 2 \phi_{a}$ in (1.112) is responsible for the opening of a gap. We search for a saddle point with a form of the variational connected Green's 
Function slightly generalized with respect to (1.84):

$$
v G_{c}^{-1}\left(q, \omega_{n}\right)=\frac{1}{\pi \bar{K}}\left(\omega_{n}^{2}+v^{2} q^{2}\right)+m^{2}+\Sigma_{1}\left(1-\delta_{n, 0}\right)+I\left(\omega_{n}\right)
$$

Where the parameter $\Sigma_{1}$ and the function $I\left(\omega_{n}\right)$ satisfy the equations (1.86) and (1.85). We give here the main steps of the solution and refer the reader to 93, 94 for more details. The variational self-energy satisfies the equation (1.87). Finally, $m$ satisfies the equation:

$$
m^{2}=\frac{4 g v}{\pi \alpha} e^{-2 \hbar \tilde{G}(0,0)}
$$

The important physical quantities are simply given such as conductivity and compressibility are simply given by $(1.15)$ and (1.55).

Since we expect the physics to be continuous for small enough $K$ (i.e. repulsive enough interactions), one can gain considerable insight by considering (see sec. 3.1) the classical limit $\hbar \rightarrow 0, K \rightarrow 0$ keeping $\bar{K}=K / \hbar$ fixed. In this limit one can solve analytically the saddle point equations (1.84)-(1.87), (1.113)-(1.114) and compute $m, \Sigma_{1}$ and $I\left(\omega_{n}\right)$. The resulting phase diagram is parameterized with two physical lengths (for $K \rightarrow 0$ ): The correlation length (or soliton size) of the pure gapped phase

$$
d=\left(\frac{4 g \bar{K}}{\alpha v}\right)^{-1 / 2}
$$

and the localization (or pinning) in the absence of commensurability length

$$
l_{0}=\left(\frac{(\alpha v)^{2}}{16 \mathcal{D} \bar{K}^{2}}\right)^{1 / 3}
$$

Contrarily to the naive direct transition predicted by the extrapolation of the RG, we find three phases 93] as shown in Figure 13. Their main characteristics in term of conductivity and compressibility are summarized in Figure 14.

Mott insulator

At weak disorder we find a replica symmetric solution with $\Sigma_{1}=0$ but with $m \neq 0$ ( $m$ depends on the disorder). $m$ is given by the simple equation:

$$
m^{2}=\frac{4 g v}{\pi \alpha} \exp \left[-\frac{\mathcal{D} \bar{K}^{1 / 2}}{\alpha^{2} \pi^{3 / 2} v^{1 / 2} m^{3}}\right] .
$$

It is convenient to work with the physical lengths $d$ and $l_{0}$ and the correlation length $\xi$ where:

$$
\xi^{2}=\frac{v^{2}}{\left(\pi \bar{K} m^{2}\right)}
$$




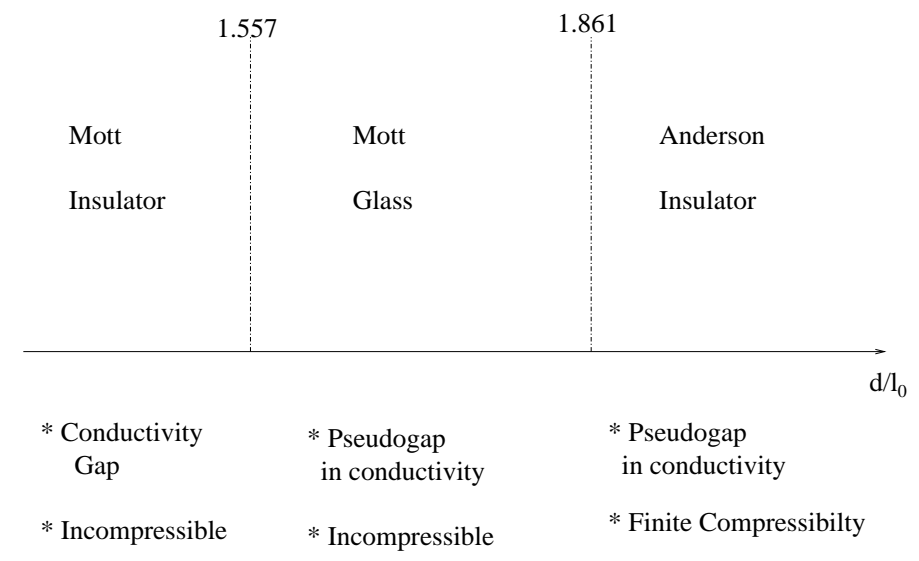

FIGURE 13. The phase diagram of a system with commensurability and disorder. $d$ is the soliton size, $l_{0}$ the localization (or pinning length)

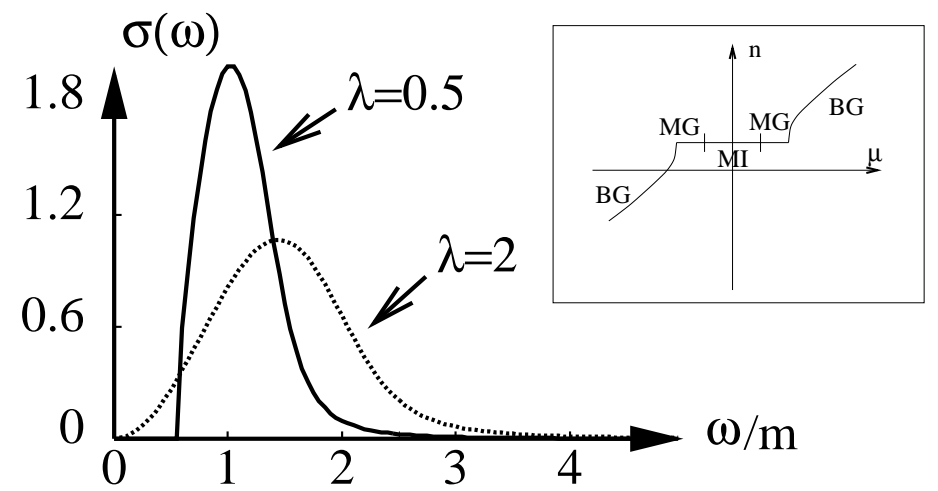

FIGURE 14. Conductivity in the Mott Insulator (solid line) and Mott Glass phases (dashed line). Insert: density $n$ versus the chemical potential $\mu$. $\lambda$ is defined in the text. 
One can then rewrite 1.117$)$ as:

$$
\frac{1}{\xi^{2}}=\frac{1}{d^{2}} \exp \left[-\frac{1}{16}\left(\frac{\xi}{l_{0}}\right)^{3}\right] .
$$

For $l_{0} / d>\frac{1}{2}\left(\frac{3 e}{4}\right)^{1 / 3},(1.119)$ has a single physical solution. For $l_{0} / d<$ $\frac{1}{2}\left(\frac{3 e}{4}\right)^{1 / 3}$, (1.119) has no solution, which means that the Mott insulator becomes unstable. In fact due to another contraint (see below) the Mott Insulator becomes unstable at an even smaller disorder.

Having $m \neq 0$ leads to zero compressibility $\kappa=0$. Disorder reduces the gap created by the commensurate potential and thus increases $\xi$ compared to the pure case. Let us now examine the equation for $I\left(\omega_{n}\right)$ giving the transport properties. An expansion around $\hbar=0$ in equation (1.85) gives the self-consistent equation for $I\left(\omega_{n}\right)$ :

$$
I\left(\omega_{n}\right)=\frac{8 \mathcal{D} v}{(\pi \alpha)^{2}} \int_{0}^{\beta \hbar} G_{c}(x=0, \tau)\left(1-\cos \left(\omega_{n} \tau\right)\right) d \tau
$$

Introducing the scaling form (to be contrasted with (1.95)):

$$
I\left(\omega_{n}\right)=m^{2} f\left(\frac{\omega_{n}}{\sqrt{\pi \bar{K} m}}\right),
$$

(1.120) can be recast in the form:

$$
f(x)=\lambda\left[1-\frac{1}{\sqrt{1+x^{2}+f(x)}}\right]
$$

where:

$$
\lambda=\frac{4 \mathcal{D} \bar{K}^{1 / 2} v}{\pi^{3 / 2} \alpha^{2} m^{3}}=\frac{1}{4}\left(\frac{\xi}{l_{0}}\right)^{3}
$$

Let us note that for $\lambda=2$, the equation 1.122 reduces to the equation (1.96). For $\lambda>2$ (1.122) has no physical solution. Using (1.123), this condition becomes:

$$
\frac{l_{0}}{d}<\frac{1}{2} e^{\frac{1}{4}}
$$

For $\lambda<2$, there is a physical solution of (1.122) such that $\lim _{x \rightarrow \pm \infty} f(x)=$ $1+\lambda$ and for $x \ll 1, f(x)=1+\alpha x^{2}+o\left(x^{2}\right)$ with $\alpha=\lambda /(2-\lambda)$. The conductivity of the Mott insulator can be obtained from $f$ in the form:

$$
\sigma(\omega)=\frac{\xi \bar{K}}{\pi} \frac{\imath x}{\left(1+f(\imath x)-x^{2}\right)}
$$

where $x=\omega / \omega^{*}$ and $\omega^{*}=v / \xi$ is the characteristic frequency associated with the correlation length $\xi$. The conductivity $\sigma(\omega)$ is zero if:

$$
\omega<\omega_{c}=\omega^{*} \sqrt{1+\lambda-3\left(\frac{\lambda}{2}\right)^{2 / 3}}
$$




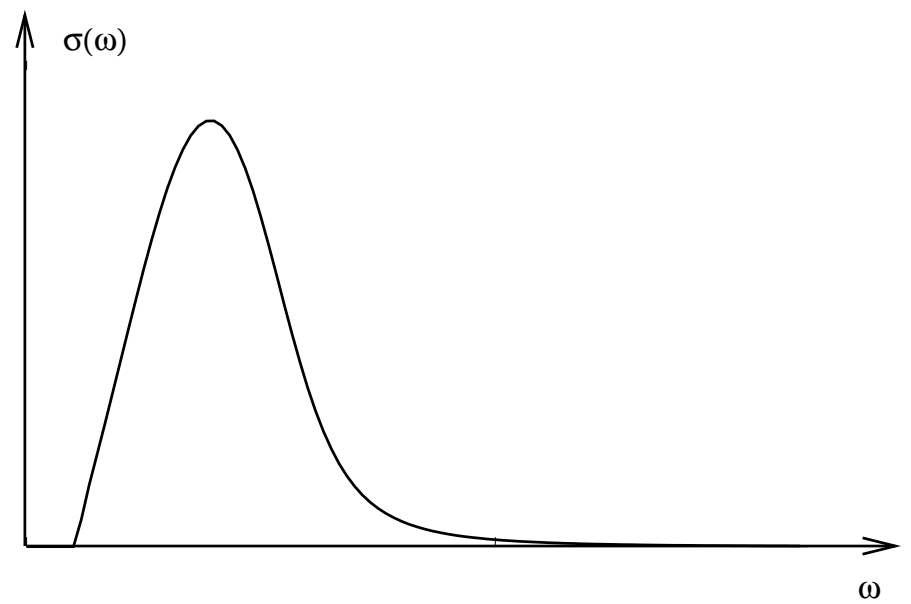

FIGURE 15. The frequency dependant conductivity of the Mott Insulator

The replica symmetric phase with $m \neq 0$ has thus a conductivity gap $\hbar \omega_{c}$ and can be assimilated to a Mott insulator (MI). The behavior of the conductivity is plotted on figure (15). Close to the threshold, one has $\sigma(\omega) \sim\left(\omega-\omega_{c}\right)^{1 / 2}$. However the gap in the conductivity $\hbar \omega_{c}$ decreases when disorder increases, and closes for $d / l_{0}=2 e^{-1 / 4} \simeq 1.557$ (see Figure 16). For $d / l_{0} \rightarrow 2 e^{-1 / 4}$, the conductivity gap vanishes linearly with $\frac{d}{l_{0}}$. For $d / l_{0}>2 e^{-1 / 4}$ the MI solution becomes unphysical even though the mass $m$ remains finite at this transition point, i.e. the system remains incompressible. For stronger disorder one must break replica symmetry, as for the pure disorder case of section 6.3. Here, however two possibilities arise depending on whether the saddle point allows for $m \neq 0$ or not. In the presence of a breaking of replica symmetry, one extra equation is needed to determine the breakpoint. As in the case of the Anderson insulator, such equation is provided by the marginality of the replicon condition discussed in section 6.4. Two phases exists:

The Anderson Glass

For large disorder compared to the commensurate potential $d / l_{0}>1.861$, $m=0$ is the only saddle point solution. The saddle point equations then reduce to those (1.84)-(1.87) . Thus, we recover the Anderson glass with interactions of section (6.3). As we have seen in section (6.3), in such phase the conductivity starts as $\sigma(\omega) \sim \omega^{2}$ showing no gap and the compressibility is finite.

In the Anderson glass phase, the disorder washes out completely the commensurate potential. The MI and the AG were the only two phases accessible by renormalization techniques 83. Within the replica variational formalism however, we find that an intermediate phase exists between them.

The Mott Glass 


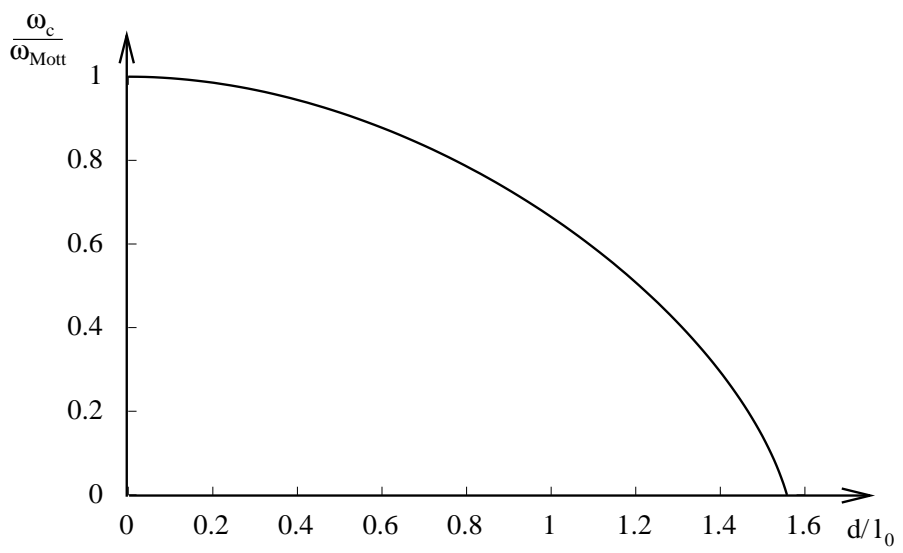

FIGURE 16. The dependance of the conductivity gap with disorder strength in the Mott insulator

For intermediate disorder $2 e^{-1 / 4}<d / l_{0}<1.861$ a phase with both $\Sigma_{1} \neq$ 0 and $m \neq 0$ is obtained. We shall call this phase the Mott Glass (MG). We shall not discuss in full detail the one-step solution of the saddle point equation here. we will rather stress the salient features of our solution. First, as a result of the marginality of replicon mode condition, $m^{2}+\Sigma_{1}$ remains constant in the MI and MG as disorder strength is increased [93, 94]. In the MG phase, $I\left(\omega_{n}\right)$ is still of the form (1.121) but $m$ is replaced by $\sqrt{m^{2}+\Sigma_{1}}$. The reduced self-energy $f(x)$ satifies (1.122) but with $\lambda=2$ in the whole Mott Glass phase. This implies that (see (1.96)) that the a.c. conductivity of the Mott Glass is identical to the one of an Anderson glass. However, since $m \neq 0$ in the MG, the system is incompressible $(\kappa=0)$ like a Mott Insulator. Thus, the Mott Glass is a new glassy phase (since it has Replica Symmetry Breaking) with characteristics intermediate between those of an Anderson Insulator and those of a Mott Insulator.

\subsection{Physical discussion}

The existence of a phase with a compressibility gap but no conductivity gap is quite remarkable since by analogy with noninteracting electrons 84 one is tempted to associate a zero compressibility to the absence of available states at the Fermi level and hence to a gap in the conductivity as well. Our solution shows this is not the case, when interactions are turned on the excitations that consists in adding one particle (the important ones for the compressibility) become quite different from the particle hole excitations that dominate the conductivity. A similar situation is obtained in the case of the one dimensional Wigner crystal [95], which has the conductivity of a perfect $1 \mathrm{~d}$ metal, $\sigma(\omega) \propto \delta(\omega)$ but a zero compressiblity since $\chi=\lim _{q \rightarrow 0} \frac{1}{\ln q}$. This argument suggest that the difference in one-particle 


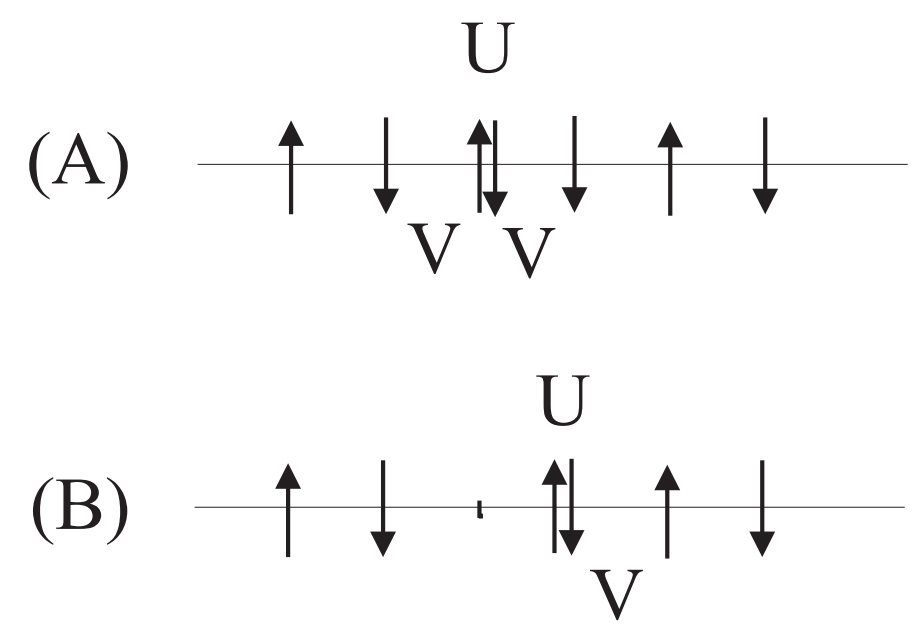

FIGURE 17. (A) Energy needed to add one particle. (B) Energy needed to make the particle hole excitations entering in the optical conductivity. Because of excitonic processes, when disorder is added the gap in the optical conductivity can close before the single particle gap. This phenonenon, leading to the MG phase, should occur regardless of the dimension.

and two-particle properties is a consequence of the strong repulsion in the system.

In addition to the variational method itself the Mott glass phase can also be obtained by two other independent methods. Higher dimensional extensions of the present problem, similar to the one made in section 6.1 can be treated around four spatial dimensions using a $d=4-\epsilon$ functional renormalization group method (totally different form the $d=2 \mathrm{RG}$ ). Such study confirms [93, 94 the existence of the intermediate Mott glass phase. One can also analyse (1.112) for zero kinetic energy and obtain the MG phase [94. Although we have done the derivation of the Mott phase for fermions in one dimension we expect its physics to survive into higher dimension. This can be seen by looking at the atomic limit (zero hoping) of an interacting fermionic system (in any dimension). If the repulsion extends over at least one interparticle distance, leading to small values of $K$, particle hole excitations are lowered in energy by excitonic effects. For example for fermions with spins with both an onsite $U$ and a nearest neighbor $V$ the gap to add one particle is $\Delta=U / 2$. On the other hand the minimal particlehole excitations would be to have the particle and hole on neighboring sites (excitons) and cost $\Delta_{\text {p.h. }}=U-V$, as shown in Figure 17. When disorder is added the gaps decrease respectively [96] as $\Delta \rightarrow \Delta-W$ and $\Delta_{\text {p.h. }} \rightarrow \Delta_{\text {p.h. }}-2 W$. Thus the conductivity gap closes, the compressibility remaining zero (for bounded disorder). According to this physical picture of the MG, the low frequency behavior of conductivity is dominated by excitons (involving neighboring sites). This is at variance from the AG 
where the particle and the hole are created on distant sites. This may have consequences on the precise low frequency form of the conductivity such as logarithmic corrections. When hopping is restored, we expect the excitons to dissociate and the MG to disappear above a critical value $K>K^{*}$. Since finite range is needed for the interactions, in all cases (fermions or bosons) $K^{*}<1$. In addition we expect $K^{*}<1 / 2$ for fermions with spins. One interesting question is the question of d.c. transport in the three phases, and whether the Mott glass has a d.c. transport closer to the Anderson or the Mott phase. Since the excitons are neutral, one simple guess would be that such excitations would not contribute to the d.c. transport. The d.c. conductivity in the Mott glass phase would thus be still exponentially activated just as in the Mott insulator. Of course more detailed studies would be needed to confirm this point.

\section{Conclusions}

Many disordered fermionic system can thus be successfully described by an elastic disordered theory. In one dimension, this situation is ubiquitous due to the importance of collective excitations. Most physical system, whether one starts with fermions, bosons or spins, can be represented in terms of bosonic excitations. In higher dimension such a description is valid in crystalline phases such as a Wigner crystal in which the quantum particles are strongly localized due to their interactions or charge density waves. Disorder then leads to rich physical phenomena coming from the competition between the elasticity, wanting a well ordered structure and the disorder that distorts the structure to gain pinning energy. This leads to the existence of many metastable configurations and to glassy properties. Using the various methods described in these notes, we now have a good description of the low energy excitations of such structures. This gives access to a host of physical properties such as the a.c. transport properties.

Clearly one of the most important open questions is the the issue of topological defects in such structures. Indeed, such defects are needed to describe the melting of these cristalline phases and will be necessary to go to more "liquid" phases in which the statistics (fermionic or bosonic) of the particles will play a much more crucial role. In addition d.c. transport is obviously dominated by such excitations. Unfortunately so far the methods able to tackle the properties in the localized phase such as the Gaussian Variational Method cannot handle such solitonic excitations, so radically new methods will need to be designed to handle them. 


\section{Acknowledgements}

The work presented in these notes results from many fruitful and enjoyable collaborations. We would like to thank R. Chitra, H. Maurey, B. S. Shastry and specially P. Le Doussal. Most importantly, nothing would have started without an initial collaboration with H. J. Schulz, to the memory of whom we would like to dedicate these lecture notes.

\section{REFERENCES}

[1] N. F. Mott and W. D. Twose, Adv. Phys. 10, 107 (1961).

[2] V. L. Berezinskii, Sov. Phys. JETP 38, 620 (1974).

[3] A. A. Abrikosov and J. A. Rhyzkin, Adv. Phys. 27, 147 (1978).

[4] E. Abrahams, P. W. Anderson, D. C. Licciardello, and T. V. Ramakrishnan, Phys. Rev. Lett. 42, 673 (1979).

[5] F. Wegner, Z. Phys. B 35, 207 (1979).

[6] K. B. Efetov, A. I. Larkin, and D. E. Khmel'nitskii, Sov. Phys. JETP 52, 568 (1980).

[7] K. B. Efetov, Adv. Phys. 32, 53 (1983).

[8] P. Nozieres, Theory of Interacting Fermi systems (W. A. Benjamin, New York, 1961).

[9] B. L. Altshuler and A. G. Aronov, in Electron-electron interactions in disordered systems, edited by A.L.Efros and M.Pollak (North-Holland, Amsterdam, 1985).

[10] A. M. Finkelstein, Z. Phys. B 56, 189 (1984).

[11] P. A. Lee and T. V. Ramakhrishnan, Rev. Mod. Phys. 57, 287 (1985).

[12] For a review see: D. Belitz and T. R. Kirkpatrick, Rev. Mod. Phys. 66261 (1994).

[13] J. Sólyom, Adv. Phys. 28, 209 (1979).

[14] V. J. Emery, in Highly Conducting One-Dimensional Solids, edited by J. T. Devreese and al. (Plenum, New York, 1979), p. 327.

[15] H. J. Schulz, in Mesoscopic quantum physics, Les Houches LXI, edited by E. Akkermans, G. Montambaux, J. L. Pichard, and J. Zinn-Justin (Elsevier, Amsterdam, 1995).

[16] J. Voit, Rep. Prog. Phys. 58, 977 (1995). 
[17] D. Senechal, this volume.

[18] P. Jordan and E. Wigner, Z. Phys. 47, 631 (1928).

[19] T. Giamarchi and H. J. Schulz, Europhys. Lett. 3, 1287 (1987).

[20] T. Giamarchi and H. J. Schulz, Phys. Rev. B 37, 325 (1988).

[21] M. P. A. Fisher, P. B. Weichman, G. Grinstein, and D. S. Fisher, Phys. Rev. B 40, 546 (1989).

[22] C. Doty and D. S. Fisher, Phys. Rev. B 45, 2167 (1992).

[23] G. Grüner, Rev. Mod. Phys. 60, 1129 (1988).

[24] H. Fukuyama, J. Phys. Soc. Jpn. 41, 513 (1976).

[25] H. Fukuyama and P. A. Lee, Phys. Rev. B 17, 535 (1978).

[26] M. V. Feigelmann and V. M. Vinokur, Phys. Lett. A 87, 53 (1981).

[27] Y. Suzumura and H. Fukuyama, J. Phys. Soc. Jpn. 52, 2870 (1983).

[28] L. P. Gorkov and I. E. Dzyaloshinski, JETP Lett. 18, 401 (1973).

[29] D. C. Mattis, J. Math. Phys. 15, 609 (1974).

[30] A. Luther and I. Peschel, Phys. Rev. Lett. 32, 992 (1974).

[31] W. Apel, J. Phys. C 15, 1973 (1982).

[32] W. Apel and T. M. Rice, J. Phys. C 16, L271 (1982).

[33] W. Apel and T. M. Rice, Phys. Rev. B 26, 7063 (1982).

[34] J. M. Kosterlitz and D. J. Thouless, J. Phys. C 6, 1181 (1973).

[35] J. M. Kosterlitz, J. Phys. C 7, 1046 (1974).

[36] C. Kane and M. P. A. Fisher, Phys. Rev. Lett. 68, 1220 (1992).

[37] C. Kane and M. P. A. Fisher, Phys. Rev. B 46, 15233 (1992).

[38] T. Giamarchi and H. Maurey, in Correlated fermions and transport in mesoscopic systems, edited by T. Martin, G. Montambaux, and J. Tran Thanh Van (Editions Frontières, Gif sur Yvette, France, 1996), cond-mat/9608006.

[39] F. D. M. Haldane, Phys. Rev. Lett. 47, 1840 (1981).

[40] T. Giamarchi and B. S. Shastry, Phys. Rev. B 51, 10915 (1995).

[41] H. Maurey and T. Giamarchi, Phys. Rev. B 51, 10833 (1995). 
[42] N. Kawakami and S. Fujimoto, Phys. Rev. B 52, 6189 (1995).

[43] E. Orignac and T. Giamarchi, Phys. Rev. B 56, 7167 (1997).

[44] E. Orignac and T. Giamarchi, Phys. Rev. B 57, 11713 (1998).

[45] V. Brunel and T. Jolicoeur, Phys. Rev. B 58, 8481 (1998).

[46] E. Orignac and T. Giamarchi, Phys. Rev. B 57, 5812 (1998).

[47] K. J. Runge and G. T. Zimanyi, Phys. Rev. B 49, 15212 (1994).

[48] G. Bouzerar, D. Poilblanc, and G. Montambaux, Phys. Rev. B 49, 8258 (1994).

[49] G. Bouzerar and D. Poilblanc, Phys. Rev. B 52, 10772 (1995).

[50] R. A. Römer and A. Punnoose, Phys. Rev. B 52, 14809 (1995).

[51] R. Berkovits and Y. Avishai, Europhys. Lett. 29, 475 (1995).

[52] S. Haas, J. Riera, and E. Dagotto, Phys. Rev. B 48, 13174 (1993).

[53] R. T. Scalettar, G. G. Batrouni, and G. T. Zimanyi, Phys. Rev. Lett. 66, 3144 (1991).

[54] S. R. White, Phys. Rev. B 48, 10345 (1993).

[55] P. Schmitteckert et al., Phys. Rev. Lett. 80, 560 (1998).

[56] M. Mézard and G. Parisi, J. de Phys. I 1, 809 (1991).

[57] T. Giamarchi and P. Le Doussal, in Statics and dynamics of disordered elastic systems, edited by A. P. Young (World Scientific, Singapore, 1998), p. 321, cond-mat/9705096.

[58] T. Giamarchi and P. Le Doussal, Phys. Rev. B 52, 1242 (1995).

[59] J. L. Cardy and S. Ostlund, Phys. Rev. B 25, 6899 (1982).

[60] P. Le Doussal and T. Giamarchi, Phys. Rev. Lett. 74, 606 (1995).

[61] M. Mézard, G. Parisi, and M. A. Virasoro, Spin Glass Theory and beyond (World Scientific, Singapore, 1987).

[62] A. I. Larkin, Sov. Phys. JETP 31, 784 (1970).

[63] A. I. Larkin and Y. N. Ovchinnikov, J. Low Temp. Phys 34, 409 (1979).

[64] There are some subtleties for systems for which the size of the particles differs from the lattice spacing. This leads to the definitions of two characteristic lengths. We ignore these differences here since they do not occur for classical CDW and 1D fermionic systems. See 57] for consequences for classical systems and [72] for the Wigner crystal. 
[65] Such a length was also obtained by Imry and Ma for the random field Ising model. So a proper acronym for this length might be the Loflim length (for Larkin-Ovchinikov, Fukuyama-Lee, Imry-Ma).

[66] This remark is originally due to S. Sachdev.

[67] T. Giamarchi and P. Le Doussal, Phys. Rev. B 53, 15206 (1996).

[68] A. Georges, O. Parcollet and S. Sachdev, cond-mat/9909239.

[69] A. L. Efros and B. I. Shklovskii, J. Phys. C 8, L49 (1975).

[70] P. A. Lee and A. I. Larkin, Phys. Rev. B 17, 1596 (1978).

[71] D. R. Nelson and V. M. Vinokur, Phys. Rev. B 48, 13060 (1993).

[72] R. Chitra, T. Giamarchi, and P. Le Doussal, Phys. Rev. Lett. 80, 3827 (1998).

[73] R. Chitra, T. Giamarchi and P. Le Doussal, to be published.

[74] A. A. Gogolin, Phys. Rep. 86, 1 (1982).

[75] A. Comtet, A. Georges, and P. Le Doussal, Phys. Lett. B 208, 487 (1988).

[76] A. O. Gogolin, A. A. Nersesyan, A. M. Tsvelik, and L. Yu, Nucl. Phys. B 540, 705 (1997).

[77] L. Balents and M. P. A. Fisher, Phys. Rev. B 56, 12970 (1997).

[78] T. P. Eggarter and R. Riedinger, Phys. Rev. B 18, 569 (1978).

[79] K. Damle, O. Motrunich, and D. A. Huse, Phys. Rev. Lett. 84, 3434 (2000).

[80] D. S. Fisher, Phys. Rev. B 50, 3799 (1994).

[81] D. S. Fisher, Phys. Rev. B 51, 6411 (1995).

[82] R. Shankar, Int. J. Mod. Phys. B 4, 2371 (1990).

[83] S. Fujimoto and N. Kawakami, Phys. Rev. B 54, R11018 (1996).

[84] M. Mori and H. Fukuyama, J. Phys. Soc. Jpn. 65, 3604 (1996).

[85] M. A. Paalanen, J. E. Graebner, R. N. Bhatt, and S. Sachdev, Phys. Rev. Lett. 61, 597 (1988).

[86] B. Grenier et al., Phys. Rev. B 57, 3444 (1998).

[87] E. Orignac and T. Giamarchi, Phys. Rev. B 567167 (1997); 575812 (1998). 
[88] M. Azuma et al., Phys. Rev. B 55, 8658 (1997).

[89] S. A. Carter et al., Phys. Rev. Lett. 77, 1378 (1996).

[90] S. Fujimoto and N. Kawakami, Phys. Rev. B 56, 9360 (1997).

[91] C. Monthus, O. Golinelli, and T. Jolicoeur, Phys. Rev. B 58, 805 (1998).

[92] T. Giamarchi, Physica B 230-232, 975 (1997).

[93] E. Orignac, T. Giamarchi, and P. L. Doussal, Phys. Rev. Lett. 83, 2378 (1999).

[94] T. Giamarchi, P. Le Doussal and E. Orignac, to be published.

[95] H. J. Schulz, Phys. Rev. Lett. 71, 1864 (1993).

[96] For small bounded disorder the gap of the Mott insulator phase is robust. 